\title{
HIGH RESOLUTION MID-INFRARED IMAGING OF ULTRALUMINOUS INFRARED GALAXIES ${ }^{1}$
}

\author{
B. T. Soifer, ${ }^{2}$ G. Neugebauer, K. MAtThews, AND E. Egami \\ Palomar Observatory, California Institute of Technology, 320-47, Pasadena, CA 91125; bts@mop.caltech.edu, gxn@mop.caltech.edu, \\ kym@mop.caltech.edu, egami@mop.caltech.edu \\ E. E. BECKLIN AND A. J. WeINBERGER \\ Department of Physics and Astronomy, University of California Los Angeles, 156205 Los Angeles, CA 90095; becklin@astro.ucla.edu, \\ alycia@astro.ucla.edu \\ M. RESSLER AND M. W. WERNER \\ Jet Propulsion Lab, 169-506, 4800 Oak Grove Drive, Pasadena, CA 91109; ressler@cougar.jpl.nasa.gov, mww@ipac.caltech.edu \\ A. S. Evans ${ }^{3}$ AND N. Z. Scoville \\ Divison of Physics, Mathematics and Astronomy, California Institute of Technology, 105-24, Pasadena, CA 91125; \\ ase@astro.caltech.edu, nzs@astro.caltech.edu \\ J. A. SURACE \\ SIRTF Science Center, California Institute of Technology, 314-6, Pasadena, CA 91125; jason@ipac.caltech.edu \\ AND \\ J. J. CONDON \\ National Radio Astronomy Observatory, 520 Edgemont Road, Charlottesville, VA 22903; jcondon@nrao.edu \\ Received 1999 September 26; accepted 1999 October 26
}

\begin{abstract}
Observations of ultraluminous infrared galaxies (ULIRGs) with an achieved resolution approaching the diffraction limit in the mid-infrared from 8 to $25 \mu \mathrm{m}$ using the Keck Telescopes are reported. We find extremely compact structures, with spatial scales of less than 0.3 (diameter) in six of the seven ULIRGs observed. These compact sources emit between $30 \%$ and $100 \%$ of the mid-infrared energy from these galaxies. We have utilized the compact mid-infrared structures as a diagnostic of whether an AGN or a compact (100-300 pc) starburst is the primary power source in these ULIRGs. In Markarian 231, the upper limit on the diameter of the $12.5 \mu \mathrm{m}$ source, 0 ."13, shows that the size of the infrared source must increase with increasing wavelength, consistent with AGN models. In IRAS 05189-2524 and IRAS $08572+3915$ there is strong evidence that the source size increases with increasing wavelength. This suggests heating by a central source rather than an extended luminosity source, consistent with the optical classification as an AGN. The compact mid-infrared sources seen in the other galaxies cannot be used to distinguish the ultimate luminosity source. If these ULIRGs are powered by compact starbursts, the star formation rates seen in the central few hundred parsecs far exceed the global rates seen in nearby starburst galaxies, and approach the surface brightness of individual clusters in nearby starburst galaxies.

Key words: galaxies: individual (Arp 220, Markarian 231, Markarian 273, UGC 5101, IRAS 05189-2524, IRAS 08572 + 3915, IRAS 17208-0014) - infrared radiation
\end{abstract}

\section{INTRODUCTION}

Ultraluminous infrared galaxies (ULIRGs; defined as those systems with luminosity from 8 to $1000 \mu \mathrm{m}$ $L_{8-1000 \mu \mathrm{m}} \geq 10^{12} L_{\odot}$ ) were discovered to be a significant class of objects in the local universe in the IRAS all sky survey (Soifer et al. 1987; Sanders et al. 1988). These systems have recently been suggested as a major component in the universe at $z>2$ through deep submillimeter surveys (cf. Lilly et al. 1999; Blain et al. 1999; Barger et al. 1998).

Whether ULIRGs are powered by dust-enshrouded AGN or starbursts has been debated since their discovery (e.g., Sanders 1999; Joseph 1999). This question is made all

\footnotetext{
${ }^{1}$ Based in part on observations obtained at the W. M. Keck Observatory which is operated as a scientific partnership among the California Institute of Technology, the University of California, and the National Aeronautics and Space Administration.

${ }^{2}$ Also at SIRTF Science Center, California Institute of Technology, 314-6, Pasadena, CA 91125.

${ }^{3}$ Current address: Department of Physics and Astronomy, SUNY Stony Brook, Stony Brook, NY 11794-3800.
}

the more important by the realization that ULIRGs could produce a major fraction of the radiant energy in the universe (Lilly et al. 1999; Heckmann 1998).

A wide variety of observational techniques have been employed to probe the nature of the central luminosity source in ULIRGS, ranging from optical spectroscopy (Sanders et al. 1988) to centimeter radio continuum imaging (Condon et al. 1991, hereafter C91), to VLBI observations (Smith, Lonsdale, \& Lonsdale 1998a) and hard X-ray observations (Rieke 1988; Nakagawa et al. 1998; Isawawa 1999). The nuclear environments of these galaxies have been effectively imaged with high spatial resolution in the optical and near-infrared (Surace et al. 1998; Surace \& Sanders 1999; Scoville et al. 1999, hereafter S99).

Observations in the thermal infrared have the potential to address the question of the origin of the luminosity by establishing the size of the regions that are radiating the infrared luminosity. The IRAS observations showed that the mid- and far-infrared luminosity in these systems emerging predominantly between 50 and $200 \mu \mathrm{m}$ was due to thermal reradiation by dust. However, the spatial resolution of those observations, $\sim 1^{\prime}-2^{\prime}$, was inadequate to place 
meaningful size constraints on the luminosity sources. Because the mid-infrared wavelengths $(8-25 \mu \mathrm{m})$ carry a significant fraction of the total bolometric luminosity in ULIRGs, ranging from $\sim 10 \%$ to more than $30 \%$ of the 8-1000 $\mu \mathrm{m}$ luminosity, observations at these wavelengths with a resolution $\leq 1^{\prime \prime}$ have the potential to constrain the sizes of the emitting regions and thereby address the nature of the underlying sources that heat the radiating dust. Observations in the $10 \mu \mathrm{m}$ atmospheric window, with a resolution $\sim 1^{\prime \prime}$, have been reported for several ULIRGs by Miles et al. (1996), and Keto et al. (1992). In most cases they found the sources to be unresolved implying that the sources were either very compact starbursts or dustenshrouded AGN.

With a diffraction limit of $0.24 \mathrm{FWHM}$ at $10 \mu \mathrm{m}$, the Keck Telescope provides a substantial improvement in spatial resolution over previous mid-infrared observations, probing the distribution of the thermal emission at the $100-300 \mathrm{pc}$ scale in nearby ULIRGs. With the introduction of mid-infrared imaging on the Keck Telescope, we have started a program to address this problem. In the first paper from this program we reported imaging of the nucleus of the closest ULIRG-Arp 220 (Soifer et al. 1999). The observations were able to spatially resolve the emission at 24.5 $\mu \mathrm{m}$ and demonstrated that the central thermal source is optically thick at this wavelength. These observations placed severe constraints on the surface brightness in the infrared luminous nuclei.

In this paper we report imaging observations from 8 to $24.5 \mu \mathrm{m}$ of a sample of the closest ULIRGs at spatial resolutions of $0.3-0$ ". 6 . These data provide the highest spatial resolution yet achieved on the thermal emission from the closest ULIRGs and trace the spatial distribution of the emergent luminosity in these systems. We adopt $H_{0}=75 \mathrm{~km} \mathrm{~s}^{-1} \mathrm{Mpc}^{-1}$.

\section{THE SAMPLE}

The objects observed were taken from the IRAS Bright Galaxy Sample (Soifer et al. 1987) with the addition of IRAS 17208-0014 (Sanders et al. 1995), which is at a low Galactic latitude. The basic information for the seven objects in the sample is given in Table 1 . The objects selected for observation were chosen on the basis of (1) meeting the luminosity definition of the ULIRGs, i.e., $L_{8-1000 \mu \mathrm{m}} \geq$ $10^{12} L_{\odot}$; (2) having a $25 \mu \mathrm{m}$ flux density greater than $1 \mathrm{Jy}$ based on IRAS measurements; (3) being at the lowest possible redshift for the best linear resolution; and (4) being available at the time of the observations. This sample contains the five closest ULIRGs in the IRAS Bright Galaxy Sample of Soifer et al., and seven of the 10 ULIRGs known with $\delta \geq-30^{\circ}$ and $z \leq 0.06$. The projected linear scales of
TABLE 1

BASIC PROPERTIES OF ULIRGs OBSERVED

\begin{tabular}{ccccc}
\hline \hline Name & $z$ & $\begin{array}{c}\log L \\
L_{\mathrm{bol}}\left[L_{\odot}\right]\end{array}$ & Spectrum & $\begin{array}{c}\text { Linear Scale } \\
\left(\mathrm{kpc} \operatorname{arcsec}^{-1}\right)\end{array}$ \\
\hline IRAS $05189-2524 \ldots \ldots$ & 0.043 & 12.08 & Sey 1 & 830 \\
IRAS $08572+3915 \ldots \ldots$ & 0.058 & 12.10 & LINER & 1200 \\
UGC $5101 \ldots \ldots \ldots \ldots \ldots$ & 0.040 & 12.00 & LINER & 820 \\
Markarian $231 \ldots \ldots \ldots \ldots$ & 0.040 & 12.52 & Sey 1 & 800 \\
Markarian $273 \ldots \ldots \ldots \ldots$ & 0.037 & 12.13 & Sey & 800 \\
Arp 220 $\ldots \ldots \ldots \ldots \ldots$. & 0.018 & 12.17 & LINER & 360 \\
IRAS $17208-0014 \ldots \ldots$ & 0.040 & 12.46 & H II & 800 \\
\hline
\end{tabular}

these objects range from 360 to $1200 \mathrm{pc} \operatorname{arcsec}^{-1}$, so that the point spread function/angular resolution of the Keck observations, 0.3 at $12 \mu \mathrm{m}$ and 0.6 at $24.5 \mu \mathrm{m}$ provide linear resolutions of $100-700 \mathrm{pc}$, depending on the object and the wavelength observed. Three of the objects in the sample have been classified as Seyfert nuclei, three as LINERS, and one as an $\mathrm{H}$ II region, based on optical spectroscopy.

\section{OBSERVATIONS AND DATA REDUCTION}

The observations were made primarily using the MIRLIN mid-infrared camera (Ressler et al. 1994) at the $\mathrm{f} / 40$ bent Cassegrain visitor port of the Keck II Telescope. The camera uses a $128 \times 128 \mathrm{Si}$ :As array with a plate scale of 0.138 pixel $^{-1}$ for a total field of view of $17^{\prime \prime} \times 17^{\prime \prime}$. Table 2 reports the central wavelengths for the filters through which each object was observed. At each wavelength the observing procedure was the same. A secondary with a square wave chop of amplitude $6^{\prime \prime}$ in the north-south or east-west direction at $4 \mathrm{~Hz}$ was employed for fast beam switching. The frames sampling each chop position were coadded separately in hardware, resulting in two images. After an interval of approximately a minute, the telescope was nodded perpendicular to the chop direction (east-west or north-south) by $6^{\prime \prime}$ and a second pair of images was obtained in order to cancel residuals in the sky and to subtract telescope emission. This procedure was repeated a number of times at each wavelength. The data were reduced by differencing the two images obtained within the chop pairs at each nod location, and then co-adding the resulting positive images, with the positions appropriately adjusted to a common location, to yield a positive image centered in a field approximately $6^{\prime \prime} \times 6^{\prime \prime}$. Because of the chopper and telescope nod spacings employed for the observations, the data are not capable of measuring flux outside a 6 " diameter region.

Observations with MIRLIN of all the ULIRGs except IRAS 05189-2524 were obtained in two nights in 1998

TABLE 2

LOG OF KeCK II Mid-INFRAREd OBSERVATIONS OF ULIRGs

\begin{tabular}{|c|c|c|c|c|c|c|c|c|}
\hline Object & $7.9 \mu \mathrm{m}$ & $8.8 \mu \mathrm{m}$ & $9.7 \mu \mathrm{m}$ & $10.3 \mu \mathrm{m}$ & $11.7 \mu \mathrm{m}$ & $12.5 \mu \mathrm{m}$ & $17.9 \mu \mathrm{m}$ & $24.5 \mu \mathrm{m}$ \\
\hline IRAS $05189 \ldots \ldots$ & & & & & & $\sqrt{ }$ & & $\sqrt{ }$ \\
\hline IRAS $08572 \ldots \ldots$ & $\sqrt{ }$ & $\sqrt{ }$ & $\sqrt{ }$ & & & $\sqrt{ } \mathbf{a}^{a}$ & $\sqrt{ }$ & \\
\hline UGC $5101 \ldots \ldots$. & & & & & & & $\sqrt{ }$ & \\
\hline Mrk $231 \ldots \ldots \ldots$ & $\sqrt{ }$ & $\sqrt{ }$ & $\sqrt{ }$ & $\sqrt{ }$ & $\sqrt{ }$ & $\sqrt{ }{ }^{a}$ & $\sqrt{ }$ & \\
\hline Mrk $273 \ldots \ldots \ldots$ & $\sqrt{ }$ & $\sqrt{ }$ & $\sqrt{ }$ & $\sqrt{ }$ & $\sqrt{ }$ & $\sqrt{ }$ & $\sqrt{ }$ & \\
\hline $\operatorname{Arp} 220 \ldots \ldots \ldots$ & $\sqrt{ }$ & $\sqrt{ }$ & $\sqrt{ }$ & $\sqrt{ }$ & $\sqrt{ }$ & $\sqrt{ }$ & $\sqrt{ }$ & $\sqrt{ }$ \\
\hline IRAS $17208 \ldots \ldots$ & $\sqrt{ }$ & & & & & $\sqrt{ }$ & & \\
\hline
\end{tabular}

${ }^{\text {a }}$ Size determination made with the Long Wavelength Spectrograph in 1999 March. 
March. The observations of the targets were interleaved with observations of nearby bright stars that were used to establish the point spread function (PSF) for the observations. The PSF stars were observed only three times per night in each of the bands at $12.5,17.9$, and $24.5 \mu \mathrm{m}$, but an attempt was made to accompany the critical observations with measurements of a nearby PSF star. At $24.5 \mu \mathrm{m}$ the PSF was always equal to the diffraction limit of the telescope to be 0"62 FWHM. At the shorter wavelengths, the size of the PSF image was set by diffraction and the pixel sampling about half of the time; at other times atmospheric seeing affected the image size.

IRAS 05189-2524 was observed in 1998 October. During this run the PSF stars were observed more frequently, but for wavelengths shorter than $\lambda \sim 17.9 \mu$ m significant variation in the measured size of the PSF star was seen.

The variation in the measured size of the PSF calibration star is the largest uncertainty in the measurement of the source sizes; this issue is addressed when observations of a particular object are described.

The MIRLIN observations were made under photometric conditions. The photometry was calibrated based on observations of four bright stars, $\alpha$ Tau, $\alpha$ Boo, $\beta$ Peg, and $\alpha$ Cet whose magnitudes, in turn, were based on IRAS photometry. The uncertainties in the photometry, based on the internal consistency of the observations, is estimated to be $5 \%$ at $\lambda \leq 17.9 \mu \mathrm{m}$ and $10 \%$ at $24.5 \mu \mathrm{m}$. The flux density corresponding to $0.0 \mathrm{mag}$ (Vega-based) was taken to follow the prescription given in the Explanatory Supplement to the IRAS Catalogs and Atlases (1989) and is given in Soifer et al. (1999).

In addition to the MIRLIN observations, UGC 5101, Markarian 231, and IRAS $08572+3915$ were observed using the Long Wavelength Spectrograph (Jones \& Puetter 1993) on the Keck I Telescope on the night of 1999 April 1. The night was not photometric and so only measurements of the sizes of the compact sources were made; all the observations were carried out at $12.5 \mu \mathrm{m}$. The chopper was set to an amplitude of $5^{\prime \prime}$ chopping north-south at a frequency $\sim 5$ Hz. Observations were made in a fashion similar to the MIRLIN observations, except that the telescope nodding was in the same direction as the chopping and the nodding amplitude was the same as the chopping amplitude. The data were reduced in a manner similar to the MIRLIN data. The pixel scale is $0^{\prime \prime} 08$ pixel $^{-1}$.

Observations of Markarian 273 were made at $3.45 \mu \mathrm{m}$ using the near-infrared camera at the Cassegrain focus of the 200 inch Hale Telescope at Palomar Observatory in 1999 May. The camera was read out in a $64 \times 64$ pixel subarray because of the high read rate required to avoid saturation. The scale is 0.125 pixel $^{-1}$. The chopper throw was $15^{\prime \prime}$ at a frequency of $\sim 1 \mathrm{~Hz}$. Observations of stars given by Elias et al. (1982) were used to establish the photometric scale.

\section{RESULTS}

The mid-infrared observations of the ULIRGs reported here show a variety of characteristics, generally consistent with their properties seen at high spatial resolution at radio wavelengths cf. C91) and in the near-infrared using NICMOS on HST (Scoville et al. 1998; S99). The midinfrared data on all the ULIRGs are presented in Figures 1, 2, and 3. In Figure 1 we present the $12.5 \mu \mathrm{m}$ images of the ULIRGs and, where the radio and near-infrared data are available, compare these maps to the equivalent maps at $8.4 \mathrm{GHz}$ from the data of C91 and to the NICMOS $2.2 \mu \mathrm{m}$ data from S99 and Scoville et al (1998). In the case of three galaxies, IRAS 05189-2524, IRAS $08572+3915$ and Markarian 231, where there is a single compact nucleus that emits a substantial fraction of the $12.5 \mu \mathrm{m}$ flux density, we have presented the corresponding PSF star at $12.5 \mu \mathrm{m}$ for comparison. In Figure 2 we compare, for the five single nucleus sources, the integrated flux at $12.5 \mu \mathrm{m}$ as a function of beam radius with the same quantity for a corresponding point source to establish the spatial distribution of the midinfrared radiation. Figure 3 displays the spectral energy distributions (SEDs) obtained from the mid-infrared data and compares these data with the corresponding IR $A S$ data and data at other wavelengths. The flux densities measured for the ULIRGs in a 4" diameter beam are presented in Table 3.

The mid-infrared observations did not establish accurate absolute positions for the sources. In some cases, described below, we use arguments regarding the similarities of the structures seen at vastly different wavelengths to argue for the physical coincidence of the sources seen at different wavelengths.

In the following sections we give results for the individual objects.

\subsection{IRAS $05189-2524$}

MIRLIN observations of IRAS $05189-2524$ were made at 12.5 and $24.5 \mu \mathrm{m}$. The shorter wavelength data have the higher signal-to-noise ratio and so are presented in Figure

TABLE 3

MIRLIN AND IRAS FLUX DENSITIES FOR ULIRGs

\begin{tabular}{|c|c|c|c|c|c|c|c|c|c|c|c|}
\hline Object & $\begin{array}{c}7.9 \mu \mathrm{m} \\
(\mathrm{Jy})\end{array}$ & $\begin{array}{c}8.8 \mu \mathrm{m} \\
(\mathrm{Jy})\end{array}$ & $\begin{array}{c}9.7 \mu \mathrm{m} \\
(\mathrm{Jy})\end{array}$ & $\begin{array}{c}10.3 \mu \mathrm{m} \\
(\mathrm{Jy})\end{array}$ & $\begin{array}{c}11.7 \mu \mathrm{m} \\
(\mathrm{Jy})\end{array}$ & $\begin{array}{c}12.5 \mu \mathrm{m} \\
(\mathrm{Jy})\end{array}$ & $\begin{array}{c}12.5 \mu \mathrm{m}^{\mathrm{a}} \\
(\mathrm{Jy})\end{array}$ & $\begin{array}{c}17.9 \mu \mathrm{m} \\
(\mathrm{Jy})\end{array}$ & $\begin{array}{c}24.5 \mu \mathrm{m} \\
(\mathrm{Jy})\end{array}$ & $\begin{array}{c}25 \mu \mathrm{m}^{\mathrm{a}} \\
(\mathrm{Jy})\end{array}$ & $\begin{array}{c}60 \mu \mathrm{m}^{\mathrm{a}} \\
(\mathrm{Jy})\end{array}$ \\
\hline IRAS $05189 \ldots \ldots$ & & & & & & 720 & 740 & & $3.2 \pm 0.2$ & 3.5 & 13.9 \\
\hline IRAS $08572 \ldots \ldots$ & $615 \pm 18$ & $434 \pm 10$ & $221 \pm 10$ & & & $218 \pm 10$ & 340 & $552 \pm 46$ & & 1.8 & 7.5 \\
\hline UGC $5101 \ldots . .$. & & & & & & $185 \pm 10$ & 260 & $256 \pm 42$ & & 1.1 & 12.1 \\
\hline Mrk $231 \ldots \ldots \ldots$ & $1370 \pm 43$ & $1488 \pm 18$ & $1081 \pm 30$ & $1075 \pm 21$ & $1355 \pm 15$ & $1836 \pm 20$ & 1930 & $3249 \pm 49$ & & 8.8 & 33.6 \\
\hline Mrk $273 \ldots \ldots \ldots$ & $267 \pm 15$ & $141 \pm 10$ & $92 \pm 10$ & $38 \pm 6$ & $150 \pm 5$ & $210 \pm 10$ & 230 & $442 \pm 31$ & & 2.3 & 23.7 \\
\hline Arp $220 \ldots \ldots \ldots$ & $521 \pm 15$ & $155 \pm 6$ & $124 \pm 14$ & $59 \pm 10$ & $175 \pm 8$ & $404 \pm 12$ & 500 & $1170 \pm 50$ & $9.8 \pm 0.2$ & 8.0 & 104.0 \\
\hline IRAS $17208 \ldots \ldots$ & $315 \pm 25$ & & & & & $202 \pm 13$ & 200 & & & 1.7 & 34.1 \\
\hline
\end{tabular}

NoTES.-The MIRLIN photometry refers to a $4 "$ diameter beam. The uncertainties listed in the table are statistical only. Photometric uncertainties in the MIRLIN data are $\pm 5 \%$ for $\lambda<20 \mu \mathrm{m}$, and $\pm 10 \%$ for $\lambda>20 \mu \mathrm{m}$. The uncertainties in the $I R A S$ data are $5 \%-10 \%$.

${ }^{\text {a }}$ Flux densities from IRAS catalog and are not color corrected. 


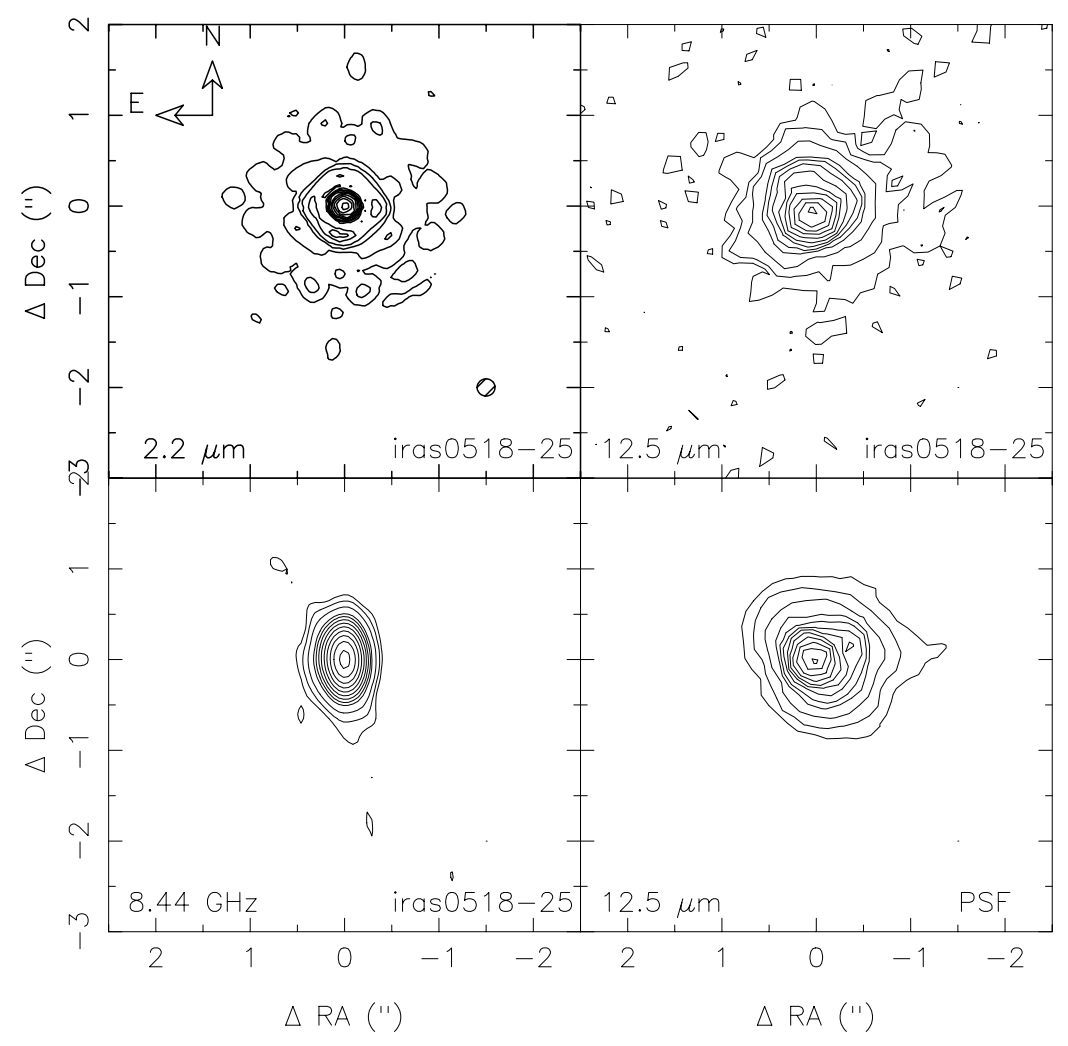

FIG. $1 a$

FIG. 1. - (a) Images of IRAS 05189 - 2524 obtained at $2.2 \mu \mathrm{m}$ (S99; upper left), $8.4 \mathrm{GHz}$ (C91; lower left), and $12.5 \mu \mathrm{m}$ (this work; upper right). The lower right panel is the PSF star image obtained at $12.5 \mu \mathrm{m}$ contemporaneously with those of IRAS $05189-2524$ and used to determine the $12.5 \mu \mathrm{m}$ size of IRAS $05189-2524$. The peak of each image is at the location 0, 0 in each panel. The contours in each panel are set so that the peak contour is $90 \%$ of the maximum surface brightness. The contours are spaced down by a factor of 1.34 so that the third contour down from the peak is at $50 \%$ of the peak surface brightness. The ninth contour down is $8.5 \%$ of the peak. Below this level the contours are spaced by a factor of 2 . The faintest contour is set at about 3 times the noise in the image. The mid-infrared data are from the MIRLIN observations. The hatched circle show the FWHM of the PSF for the $2.2 \mu \mathrm{m}$ observations. (b) Images of IRAS $08572+3915$. The format, source locations and contour levels are the same as in $(a)$. The mid-infrared data are from the LWS observations. The $2.2 \mu \mathrm{m}$ and $8.4 \mathrm{GHz}$ data are from S99 and C91, respectively. The hatched circles show the FWHM of the PSF for the appropriate observations. (c) Images of UGC 5101. The format is $2.2 \mu \mathrm{m}$ image (S99) (left), $12.5 \mu \mathrm{m}$ image (center), $8.4 \mathrm{GHz}$ image (C91) (right). The source locations and contours are set as in $(a)$. The mid-infrared data are from the MIRLIN observations. The $2.2 \mu \mathrm{m}$ and $8.4 \mathrm{GHz}$ data are from S99 and C91, respectively. The hatched circles show the FWHM of the PSF for the observations. (d) Images of Markarian 231. The format is $12.5 \mu \mathrm{m}$ image (left), $12.5 \mu \mathrm{m}$ PSF star (center), $8.4 \mathrm{GHz}$ image (right). The source locations and contours are set as in Figure 1a. The mid-infrared data are from the LWS observations. The 8.4 GHz data are from C91. The hatched circle in the $8.4 \mathrm{GHz}$ panel shows the FWHM of the PSF for this observation. (e) Images of Markarian 273. The format, source locations and contour levels are the same as in $(c)$. The mid-infrared data are from the MIRLIN observations. The $2.2 \mu \mathrm{m}$ and $8.4 \mathrm{GHz}$ data are from S99 and C91, respectively. The plus symbols are at the same coordinates in each panel and are centered on the peaks of the $2.2 \mu \mathrm{m}$ image. There is excellent agreement between the locations of the 2.2 and $12.5 \mu \mathrm{m}$ peaks. The discrepancy between the positions of the infrared peaks and the radio peak is real (see text). The hatched circles show the FWHM of the PSF for the observations. $(f)$ Images of Arp 220. The format, source locations and contour levels are the same as in $(c)$. The mid-infrared data are from the MIRLIN observations and are from Soifer et al. (1999). The 2.2 and $8.4 \mathrm{GHz}$ data are from S99 and C91, respectively. The hatched circle in the $12.5 \mu \mathrm{m}$ panel shows the FWHM of the PSF for the observation. $(g)$ Images of IRAS $17208-0014$. The left image is $2.2 \mu \mathrm{m}$, the right image is $12.5 \mu \mathrm{m}$. The source locations and contour levels are the same as in Figure $1 a$. The mid-infrared data are from the MIRLIN observations. The $2.2 \mu \mathrm{m}$ data are from S99. The hatched circles show the FWHM of the PSF for the observations.

1. Images at $12.5 \mu \mathrm{m}$ (MIRLIN), $2.2 \mu \mathrm{m}$ (NICMOS; S99) and $8.4 \mathrm{GHz}(\mathrm{VLA}$; $\mathrm{C} 91)$ are shown in Figure $1 a$. The dominant central source is clear in all three maps. At $8.4 \mathrm{GHz}$, the single source is concentrated with a measured size of $0^{\prime \prime} .2 \times 0.17$ (C91). At $2.2 \mu \mathrm{m}$, the unresolved source is less than 0.2 and contains $\sim 75 \%$ of the total flux measured in a $5^{\prime \prime}$ diameter beam, and $70 \%$ of the flux contained in an $11^{\prime \prime}$ diameter beam (S99).

The source appears virtually unresolved in the MIRLIN image. As can be seen from Figure 2, there is excellent agreement between the curve of growth for IRAS 05189-2524 and the PSF star observation. The apparent size of the PSF star varied during the time of the observations, so the limit on the size of IRAS 05189-2524 is not as stringent as would otherwise be achieved. At 12.5 $\mu \mathrm{m}$ the size is less than $0 " 2$ (Table 4). The source size at $24.5 \mu \mathrm{m}$ is an upper limit as well and is larger and thus consistent with that established at $12.5 \mu \mathrm{m}$.

As seen in Figure 3 and Table 3, the flux densities measured by MIRLIN at 12.5 and $24.5 \mu \mathrm{m}$ agree well with the IRAS observations of this source; virtually all of the luminosity of this source at 12.5 and $24.5 \mu \mathrm{m}$ is contained within the unresolved source measured here.

The MIRLIN observations of IRAS $05189-2524$ lead to a limit on the source diameter of less than $170 \mathrm{pc}$ in the mid-infrared (Table 5). The combination of flux density and upper limit on the source sizes (Table 4), imply the brightness temperatures $T_{b}>123 \mathrm{~K}$ and $T_{b}>85 \mathrm{~K}$ at 12.5 and $24.5 \mu \mathrm{m}$, respectively. The flux ratio between 12.5 and $24.5 \mu \mathrm{m}$ corresponds to a color temperature $T_{c}=169 \mathrm{~K}$, 


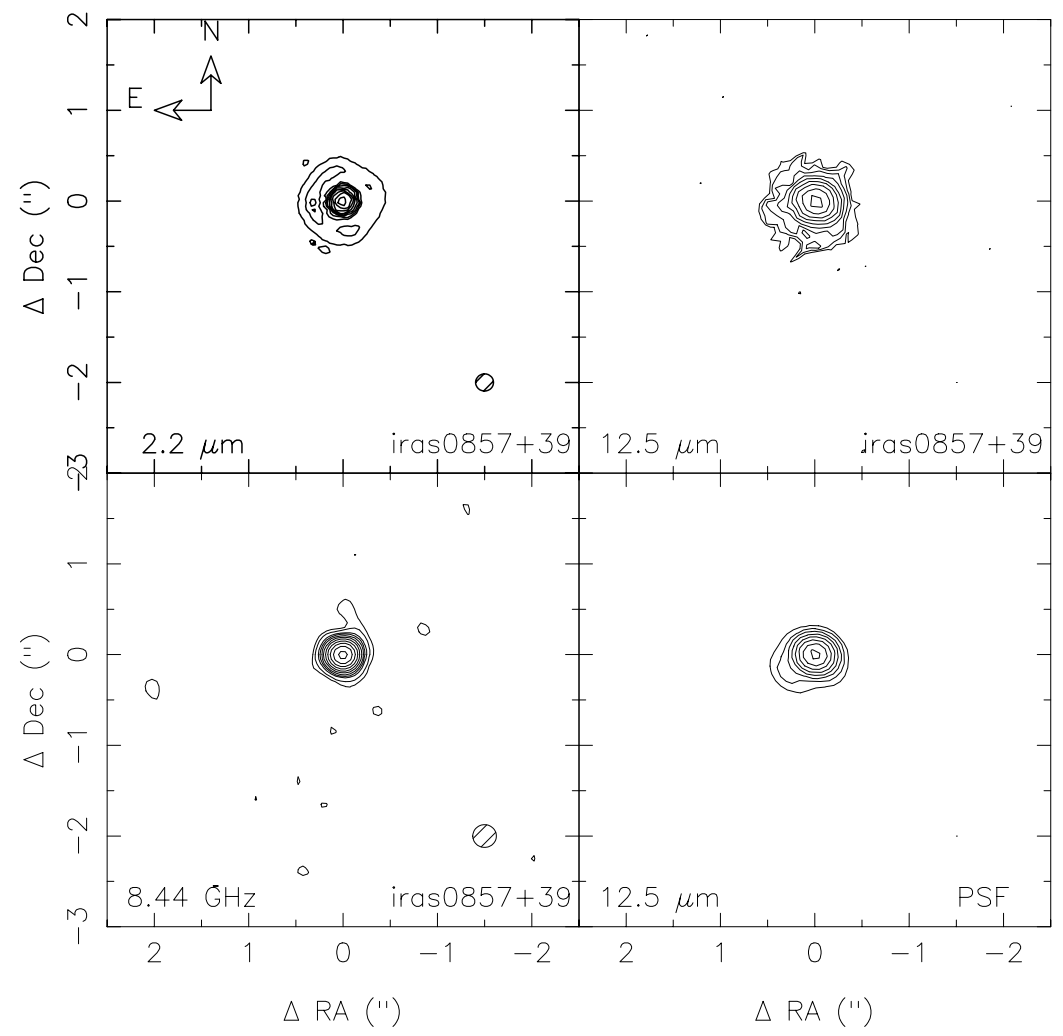

FIG. $1 b$

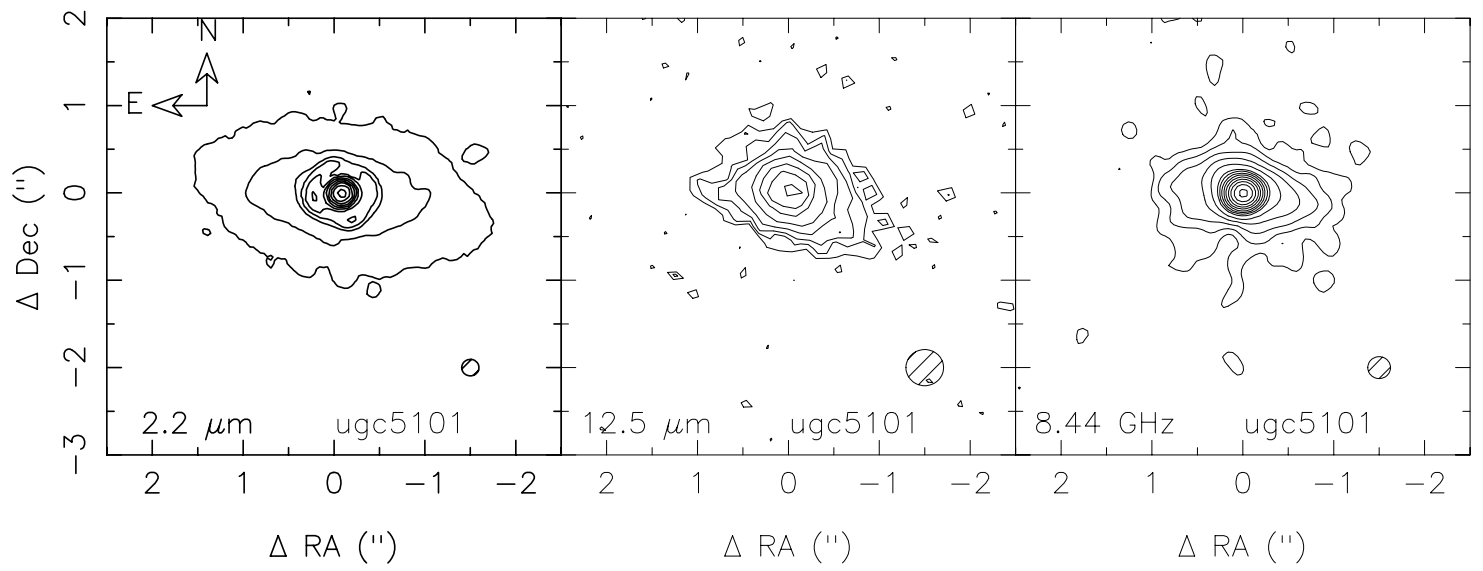

FIG. $1 c$

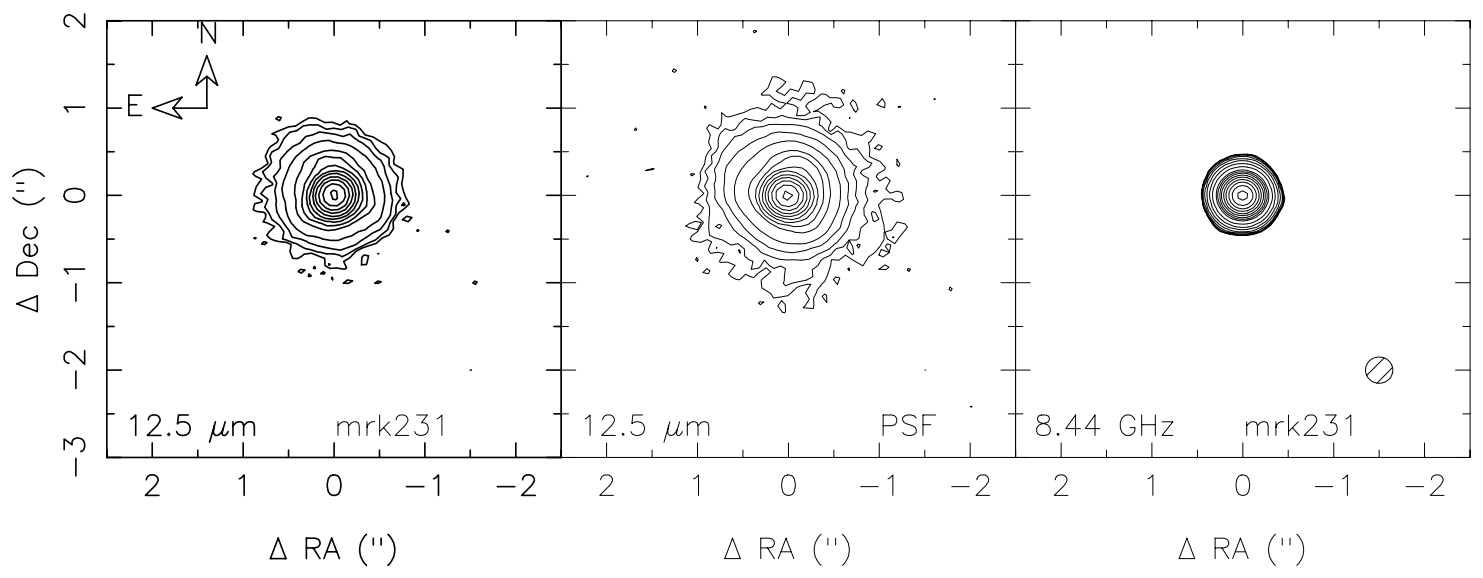

FIG. $1 d$ 


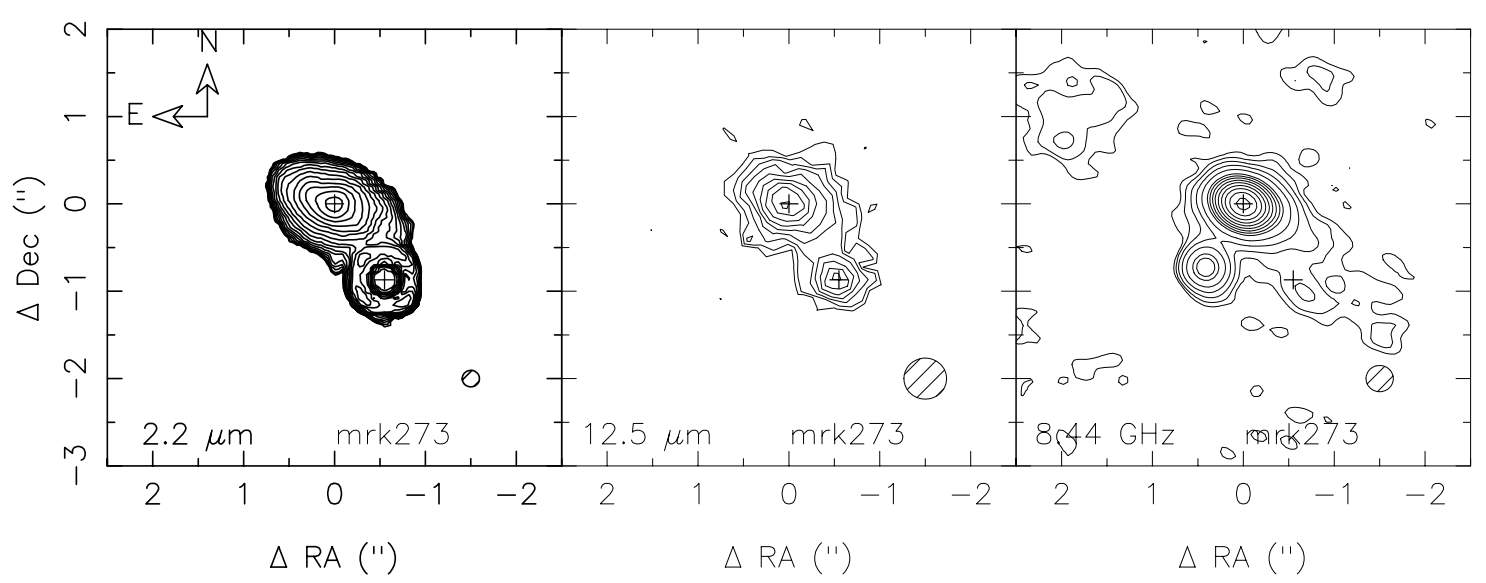

FIG. $1 e$

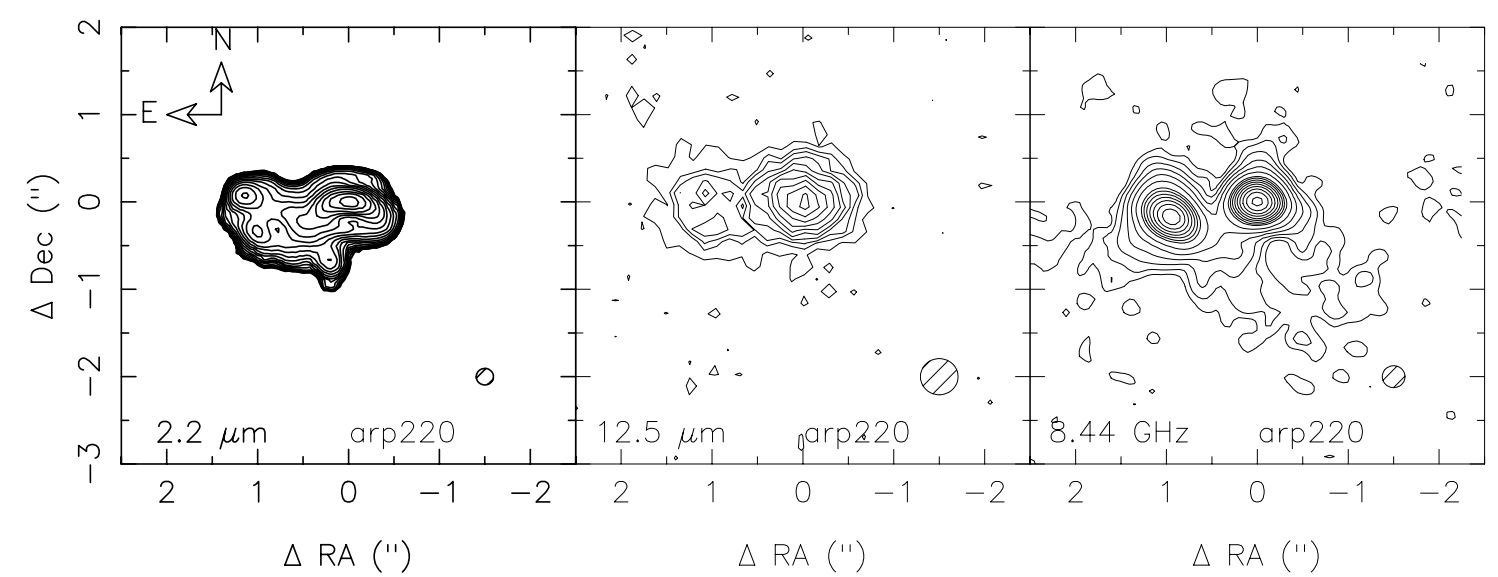

FIG. $1 f$

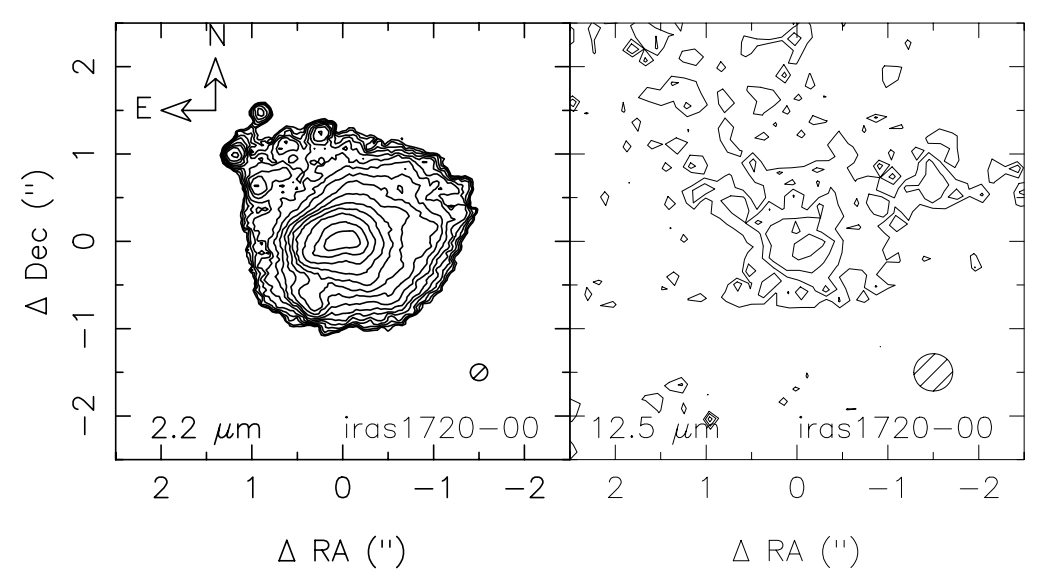

FIG. $1 g$

while the color temperature derived from the 25 and $60 \mu \mathrm{m}$ IRAS measurements is $83 \mathrm{~K}^{4}$

A central source with the bolometric luminosity of IRAS $05189-2524$ would heat silicate or graphite grains to the

${ }^{4}$ The brightness temperature, $T_{b}$, is defined by $f_{v}=\Omega \times B_{v}\left(T_{b}\right)$, where $B_{v}\left(T_{b}\right)$ is the blackbody emission at frequency $v$ for temperature $T_{b}$ and $\Omega$ is the solid angle subtended by the source. The color temperature of a source, $T_{c}$, measured between frequencies $v_{1}$ and $v_{2}$ is found from $f_{v_{1}} / f_{v_{2}}=$ $\left[B_{v_{1}}\left(T_{c}\right)\right] /\left[B_{v_{2}}\left(T_{c}\right)\right]$. apparent color temperature between 12 and $24.5 \mu \mathrm{m}, T_{c} \sim$ $170 \mathrm{~K}$, at a radius of $30-60 \mathrm{pc}$ using the grain parameters of Draine \& Lee (1984). This is much smaller than the observational limit. Alternatively, the size of an optically thin source which produces the $25-60 \mu \mathrm{m}$ flux ratio with a color temperature of $83 \mathrm{~K}$ is significantly larger, $170-250 \mathrm{pc}$ in radius, again using the Draine \& Lee grain parameters.

\subsection{IRAS $08572+3915$}

MIRLIN observations of IRAS $08572+3915$ were obtained at five wavelengths (Table 2). In addition, LWS 

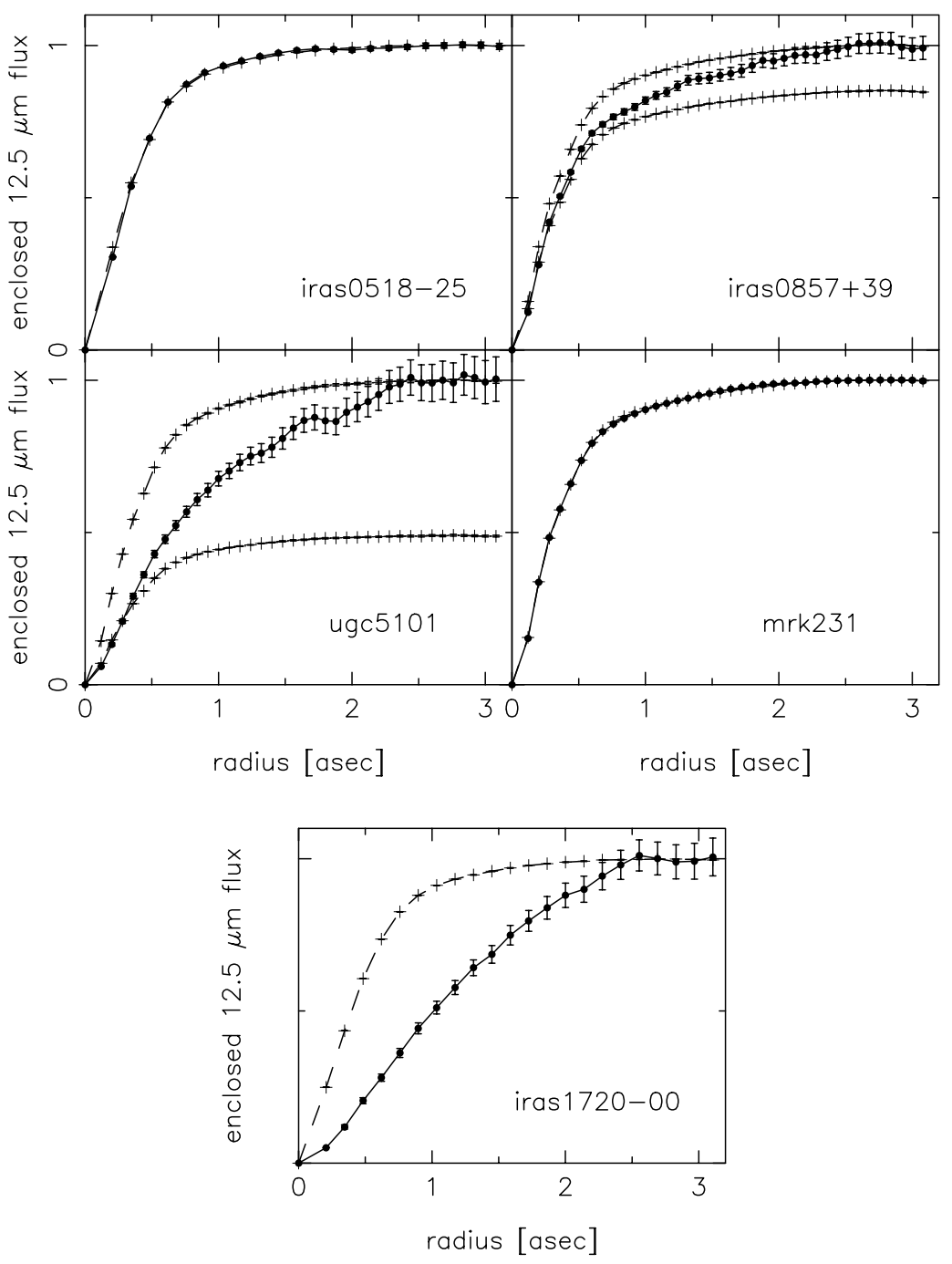

FIG. 2. - Curves of growth for the objects (solid line) and PSFs (dashed line) for the five ULIRGS which are single nuclei. In all five the PSFs have been normalized so the enclosed flux of the PSF within a radius of 2".5 equals that of the object. For the objects, the sky level was set to be the value in an annulus between 2".5 and 3". In the plots for IRAS $08572+3915$ and UGC 5101 two normalizations are used for the PSF; in the lower curve the PSF is set to match the central profile of the galaxy, while the upper curve matches the enclosed flux from the PSF and the galaxy at the outer radius of the measurement beams, as for the other galaxies. The data of IRAS $0857+39$ and Markarian 231 were obtained with LWS as outlined in the text; the other three data sets are from MIRLIN observations.

TABLE 4

Properties of COMPaCt SOURCES IN ULIRGs

\begin{tabular}{|c|c|c|c|c|c|c|c|c|}
\hline \multirow[b]{2}{*}{ ОвЈеСт } & \multicolumn{2}{|c|}{$12.5 \mu \mathrm{m}$} & \multicolumn{2}{|c|}{$17.9 \mu \mathrm{m}$} & \multicolumn{2}{|c|}{$24.5 \mu \mathrm{m}$} & \multirow{2}{*}{$\begin{array}{c}8.4 \mathrm{GHz} \\
\begin{array}{c}\text { Size } \\
(\operatorname{arcsec})\end{array}\end{array}$} & \multirow{2}{*}{$\frac{2.2 \mu \mathrm{m}}{\begin{array}{c}\text { Size } \\
(\operatorname{arcsec})\end{array}}$} \\
\hline & $\begin{array}{l}\% \text { Flux }^{\mathrm{a}} \\
\text { in core }\end{array}$ & $\begin{array}{c}\text { Size } \\
(\operatorname{arcsec})\end{array}$ & $\begin{array}{l}\% \text { Flux }^{\mathrm{a}} \\
\text { in core }\end{array}$ & $\begin{array}{c}\text { Size } \\
(\operatorname{arcsec})\end{array}$ & $\begin{array}{l}\% \text { Flux }^{\mathrm{a}} \\
\text { in core }\end{array}$ & $\begin{array}{c}\text { Size } \\
(\operatorname{arcsec})\end{array}$ & & \\
\hline IRAS $05189 \ldots \ldots$ & 100 & $<0.20$ & $\ldots$ & $\ldots$ & 100 & $<0.26$ & $0.20 \times 0.17$ & $<0.2$ \\
\hline IRAS $08572 \ldots \ldots$ & $\sim 80$ & $<0.22^{\mathrm{b}}$ & $\sim 100$ & $<0.25$ & $\ldots$ & $\ldots$ & $0.09 \times 0.07$ & $<0.2$ \\
\hline UGC $5101 \ldots \ldots$. & 50 & $<0.25$ & 60 & $<0.25$ & $\ldots$ & $\ldots$ & $0.14 \times 0.11$ & $<0.2$ \\
\hline Mrk $231 \ldots \ldots \ldots$ & 100 & $<0.13$ & 85 & $<0.25$ & $\ldots$ & $\ldots$ & $\leq 0.07 \times 0.06$ & $<0.2$ \\
\hline Mrk 273 N......... & 67 & $<0.3$ & $\ldots$ & $\ldots$ & $\ldots$ & $\ldots$ & $0.32 \times 0.18$ & $\sim 0.3$ \\
\hline Mrk 273SW ...... & 50 & $<0.3$ & $\ldots$ & $\ldots$ & $\ldots$ & $\ldots$ & $0.18 \times 0.11$ & $<0.2$ \\
\hline Arp 220 E ........ & $\ldots$ & $\ldots$ & $\ldots$ & $\ldots$ & 25 & $\ldots$ & $0.32 \times 0.19$ & $<0.2$ \\
\hline Arp $220 \mathrm{~W} \ldots \ldots$ & $\ldots$ & $\ldots$ & $\ldots$ & $\ldots$ & 75 & 0.40 & $0.21 \times 0.14$ & 0.4 \\
\hline IRAS $17208 \ldots \ldots$ & 100 & 2.0 & $\ldots$ & $\ldots$ & $\ldots$ & $\ldots$ & $0.26 \times 0.32^{\mathrm{c}}$ & 2 \\
\hline
\end{tabular}

a Percentage of flux measured in 4" diameter beam.

b Size measurement from LWS observations.

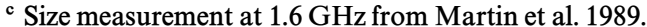



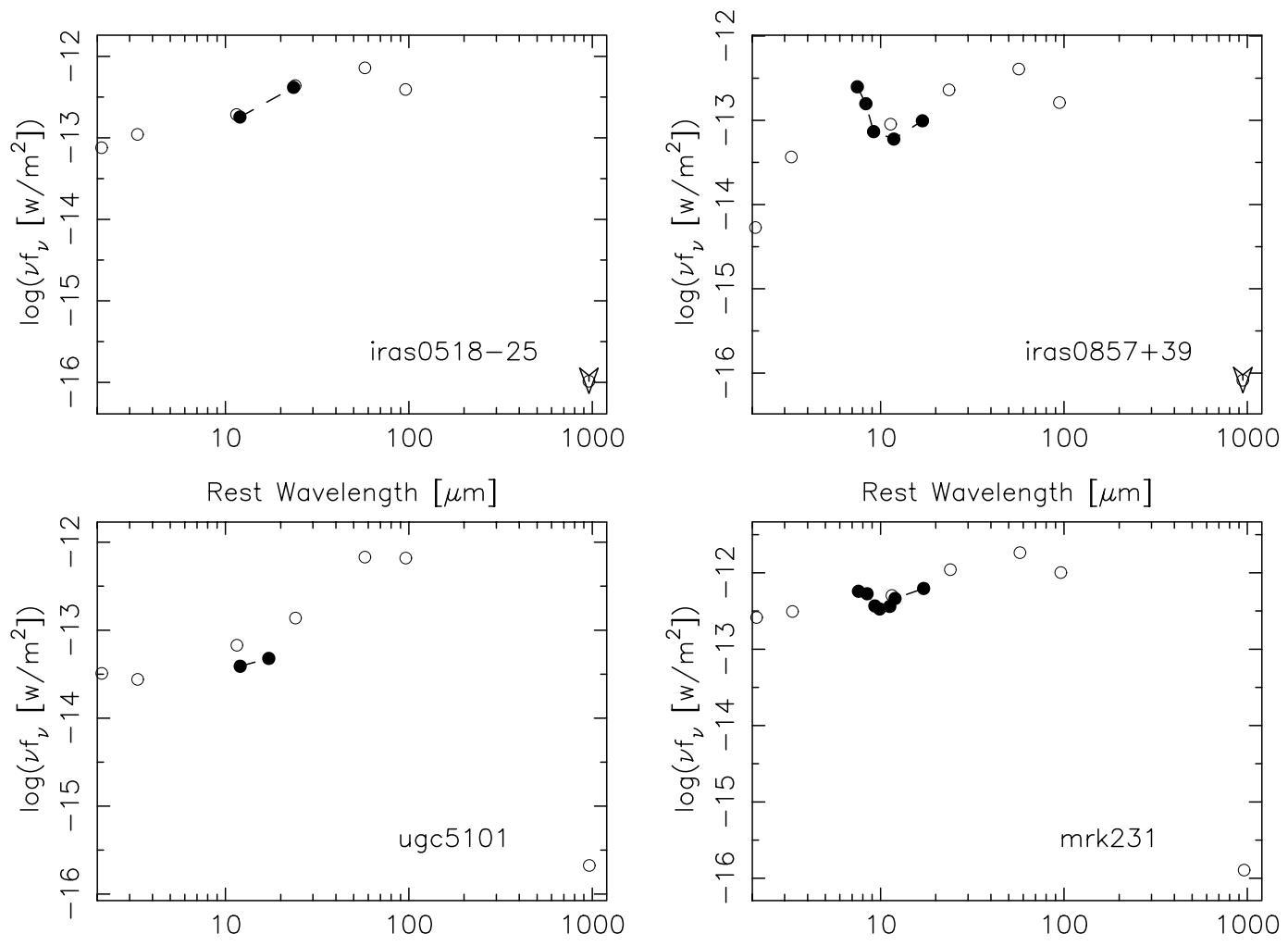

Rest Wavelength $[\mu \mathrm{m}]$

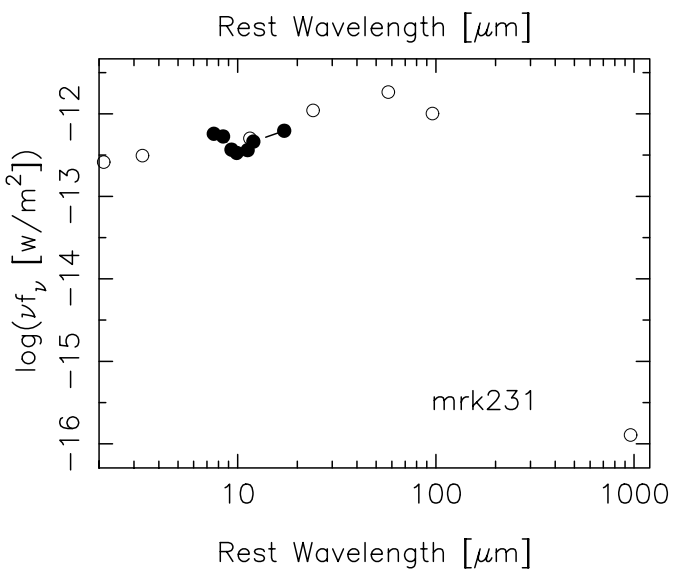

FIG. $3 a$
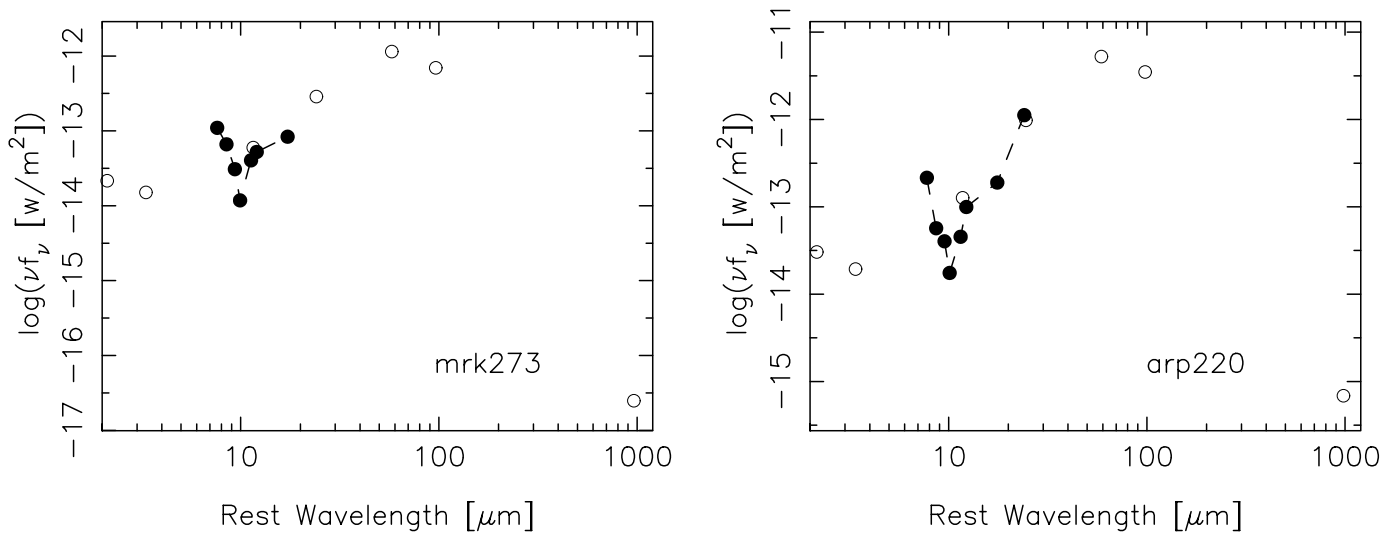

Rest Wavelength $[\mu \mathrm{m}]$

Rest Wavelength $[\mu \mathrm{m}]$

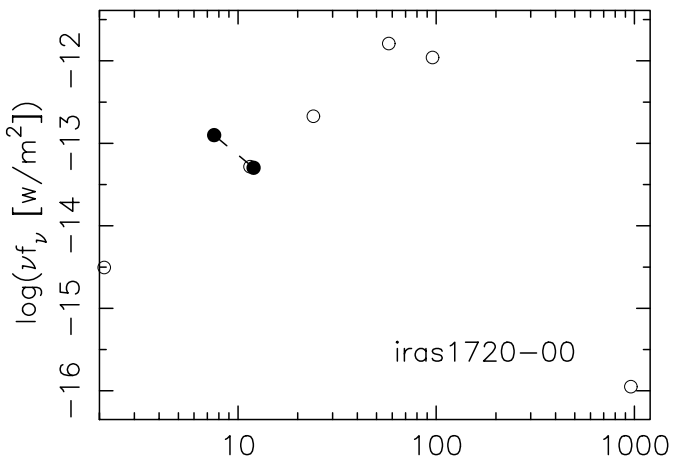

Rest Wavelength $[\mu \mathrm{m}]$

FIG. $3 b$

FIG. 3.-Spectral energy distributions of the seven ULIRGS studied are shown as a function of the rest wavelength from $\sim 1 \mu \mathrm{m}$ to $1 \mathrm{~mm}$. Filled circles represent the MIRLIN data, while open circles have been taken from Carico et al. (1988) (near-infrared data, 5" diameter beam) and Soifer et al. (1989) (IRAS data $\sim 1^{\prime}$ diameter beam). The MIRLIN photometry has been calculated for a $4^{\prime \prime}$ diameter beam centered on the object or between the two nuclei if multiple nuclei are present. As discussed in the text, the photometry is based on IRAS standards. 
TABLE 5

INFRARED SIZES OF ULRIGs

\begin{tabular}{|c|c|c|c|c|}
\hline \multirow[b]{2}{*}{ ОвЈест } & \multicolumn{2}{|c|}{ SIzE OF MID-IR CORE } & \multicolumn{2}{|c|}{$\begin{array}{c}L / A \\
\left(L_{\odot} \mathrm{kpc}^{-2}\right)\end{array}$} \\
\hline & $(\operatorname{arcsec})$ & (pc) & $\operatorname{Max}$ & Min \\
\hline IRAS $05189 \ldots \ldots$ & $<0.2$ & $<166$ & $5.6 \times 10^{13}$ & $2.1 \times 10^{13}$ \\
\hline IRAS $08572 \ldots \ldots$ & $<0.22$ & $<260$ & $>2.8 \times 10^{13}$ & $>6.0 \times 10^{12}$ \\
\hline UGC $5101 \ldots . .$. & $<0.25$ & $<205$ & $>1.1 \times 10^{13}$ & $>1.0 \times 10^{12}$ \\
\hline Mrk $231 \ldots \ldots \ldots$ & $<0.13$ & $<104$ & $3.9 \times 10^{14}$ & $1.0 \times 10^{14}$ \\
\hline Mrk 273 N ....... & $<0.3$ & $<240$ & $>2.2 \times 10^{13}$ & $>1.0 \times 10^{12}$ \\
\hline Arp $220 \ldots$ & 0.40 & 140 & $6.0 \times 10^{13}$ & $1.2 \times 10^{13}$ \\
\hline IRAS $17208 \ldots \ldots$ & 2.0 & 1600 & $1.2 \times 10^{12}$ & $3.0 \times 10^{10}$ \\
\hline
\end{tabular}

observations of IRAS $08572+3915$ were obtained at $12.5 \mu \mathrm{m}$. Optical and near-infrared imaging (Sanders et al. 1988; Carico et al. 1990; S99) show two galaxies separated by about $5^{\prime \prime}$ in a northwest-southeast orientation. The southeast source is not detected in the radio (C91) or in the present data.

The $12.5 \mu \mathrm{m}$ LWS image in Figure $1 b$ shows a bright central source (the northwest source in the near-infrared) that is unresolved. The size limit at $17.9 \mu \mathrm{m}$ is consistent with that found at $12.5 \mu \mathrm{m}$. Figure $1 b$ and Figure 2 show that the central source is dominant in the mid-infrared and radio, containing $\sim 80 \%$ of the total flux measured in a $4 "$ diameter beam. At $2.2 \mu \mathrm{m}$, the central unresolved source contains $\sim 60 \%$ of the total flux in an 11".4 diameter beam (S99).

The most stringent observations constraining the size and spatial distribution of the source are the LWS observations at $12.5 \mu \mathrm{m}$. Five observations of the PSF star (BS3275) were interleaved with eight observations of the object. The average FWHM of the object $\left(00^{\prime \prime} 39 \pm 0\right.$ " 04$)$ was marginally greater than the average FWHM of the PSF $\left(0,34 \pm 0^{\prime \prime} 01\right)$. Formally this leads to a size of the $12.5 \mu \mathrm{m}$ source of 0 "' 19 , but this is only a $1 \sigma$ result. A $3 \sigma$ limit on the size of the compact source is $\sim 0.3$. As illustrated in Figure 2 , there appears to be a measurable flux, approximately $20 \%$ of the total at $12.5 \mu \mathrm{m}$, that extends beyond the central core and is contained within $4 "$. The figure shows clearly that the flux measured in IRAS $08572+3915$ continues to increase beyond the beam where the flux in the PSF star has stopped increasing.

Figure 3 and Table 3 compare the Keck photometry in a 4" diameter beam for IRAS $08572+3915$ with the IRAS measurements. The MIRLIN photometry accounts for $70 \%$ of the IRAS flux density at $12.5 \mu \mathrm{m}$. The MIRLIN observations were taken with comparatively narrow filters, i.e., $\Delta \lambda / \lambda<\sim 0.1$, from 7.9 to $17.9 \mu \mathrm{m}$, while the $\operatorname{IRAS} 12 \mu \mathrm{m}$ filter bandpass extends from 8-15 $\mu \mathrm{m}$. Given the uncertainties in the corrections for the different bandpasses, the MIRLIN data are consistent with all the flux detected by $I R A S$ being confined to a $4^{\prime \prime}$ diameter beam surrounding the detected source although it is possible that some of the flux could be accounted for by extended emission associated with the northwest source or from the southeastern source.

Neither the MIRLIN nor the LWS observations detected the galaxy approximately $5^{\prime \prime}$ southeast of the object centered in Figure $1 b$. The MIRLIN data limit the contribution of the southeastern source to less than $30 \%$ of the total 12.5 $\mu$ m flux; it is likely that the southeast galaxy does not contribute significantly to the total infrared luminosity in this system. This is consistent with the optical and near-infrared morphology and colors of this galaxy, which are indicative of quiescent galaxy nuclei (Surace et al. 1998; Surace \& Sanders 1999).

\section{3. $U G C 5101=I R A S 09321+6134$}

MIRLIN observations of UGC 5101 were obtained at 12.5 and $17.9 \mu \mathrm{m}$. Contour plots of this source in Figure $1 c$ show that at all wavelengths the structure is basically the same: a bright central core centered on an apparently elliptical structure having a major axis at a position angle of $\sim 80^{\circ}$. While the position angle of the elliptical structures agree well at $2.2 \mu \mathrm{m}$ and $8.4 \mathrm{GHz}$, there is a suggestion that the position angle of the ellipse at $12.5 \mu \mathrm{m}$ is $\sim 15^{\circ}$ smaller. At $8.4 \mathrm{GHz}$ the central source contains $80 \%$ of the radio flux (C91), while at $2.2 \mu \mathrm{m}$, the unresolved peak contains $35 \%$ of the flux in a $5^{\prime \prime}$ diameter beam (S99).

As shown in Figure 2, at $12.5 \mu \mathrm{m}$ approximately half of the total flux measured in a 4 " diameter beam is contained in the central compact source. The inner central compact source is indistinguishable from the PSF (Fig. 2). From the dispersion in the sizes determined for the PSF star (BS3775) the compact source has a size $(3 \sigma)$ less than 0 "'.22. The size measured at $17.9 \mu \mathrm{m}$ is consistent with that measured at $12.5 \mu \mathrm{m}$.

The data of Figure 3 and Table 3 show that at $12.5 \mu \mathrm{m}$ the flux measured by MIRLIN is $\sim 60 \%$ of that measured by IRAS. This suggests that there is low surface brightness emission extended on a larger scale than $4^{\prime \prime}$. The $12.5 \mu \mathrm{m}$ flux density contained in the compact source is $\sim 30 \%$ of the total flux density at $12 \mu \mathrm{m}$ measured by IRAS.

\subsection{Markarian $231=U$ UC $8058=I R A S 12541+5708$}

Markarian 231 has long been known both as an infrared luminous system (Rieke \& Low 1972) and a Seyfert 1 system (Boksenberg et al. 1977). The IRAS survey found that it is the most luminous object within $300 \mathrm{Mpc}$, with a bolometric luminosity of $3 \times 10^{12} L_{\odot}$.

MIRLIN images of Markarian 231 were obtained at seven wavelengths from 7.9 to $17.9 \mu \mathrm{m}$. In addition, LWS imaging was obtained at $12.5 \mu \mathrm{m}$. Figure $1 d$ shows contour plots of Markarian 231 at $12.5 \mu \mathrm{m}$ from the LWS data and data at $8.4 \mathrm{GHz}(\mathrm{C} 91)$. The emission at all the mid-infrared wavelengths is dominated by the very bright central source and is effectively unresolved. The radio continuum is entirely from the bright nucleus.

LWS imaging was used to establish the size of the central source at $12.5 \mu \mathrm{m}$. The LWS observations consisted of 11 measurements of the size of Markarian 231, interleaved 
with nine measurements of a near-by PSF star (BS 4905) over a period of $2 \mathrm{hr}$. The average FWHM of the PSF star and Markarian 231 were indistinguishable and were slightly greater than the diffraction limit of the telescope and the rms (population) scatter in the FWHM was 0"04. The central source thus is unresolved in these observations. We have chosen to set a $3 \sigma$ limit on its FWHM of 0 ".13 on the basis that a difference 3 times the average standard deviation of the FWHM would have been easily detected.

The SED of Markarian 231 is plotted in Figure 3 and shows clearly that all the flux measured by $I R A S$ is detected in the 4 " diameter beam. The SED shows a dip at $10 \mu \mathrm{m}$ apparently due to foreground silicate absorption of the central source.

\subsection{Markarian $273=U G C 8696=I R A S 13428+5608$}

The MIRLIN observations of Markarian 273 consist of imaging at seven wavelengths from 7.9 to $17.9 \mu \mathrm{m}$. Figure $1 e$ shows a comparison of the MIRLIN $12.5 \mu \mathrm{m}$ data with the 8.4 GHz map (C91) and the NICMOS $2.2 \mu$ m image (S99), while a montage of images of Markarian 273 is shown in Figure 4. At $12.5 \mu \mathrm{m}$ the source structure is basically double, with the northern source brighter than the southwestern source at most wavelengths. A similar morphology is seen at $2.2 \mu \mathrm{m}$ (S99), but not in the radio (C91). In comparing the $12.5 \mu \mathrm{m}$, NICMOS and $8.4 \mathrm{GHz}$ data we have assumed the spatial coincidence between the bright northern source in each image. The overall agreement between the $12.5 \mu \mathrm{m}$ and NICMOS images is excellent, while the discrepancy between the positions of the southwestern source in the infrared and radio is real. The position angle between the northern and southwestern sources is the same (to within the uncertainties) in the $12.5,2.2$, and $8.4 \mathrm{GHz}$ images, while the source separations are 1".0, 1".0, and $1^{\prime \prime} .2$ at these same wavelengths. The apparent separation between the infrared and radio sources, $0^{\prime \prime} .2$, corresponds to $160 \mathrm{pc}$ at the distance of Markarian 273. Note also that the flux ratio between the northern and southwestern source is considerably different between the infrared and radio.

As shown in Figure 4, the structure seen in Markarian 273 at all infrared wavelengths from 3.4 to $17.9 \mu \mathrm{m}$ is basically the same. There is no evidence for a mid-infrared counterpart to the southeastern source seen at $8.4 \mathrm{GHz}$ by C91 (Fig. 1e) and at $5 \mathrm{GHz}$ by Knapen et al. (1997). The bright source present to the southeast in the radio map is suggested to be a background radio object by Knapen et al., although the statistical likelihood of a background source falling within $1^{\prime \prime}$ of the location of the radio core of Markarian 273 is less than $10^{-5}$. There is a faint compact object found in the NICMOS images (S99) at the location of southeast radio source, although there is no evidence for any mid-infrared emission at this location. The upper limit on the $12.5 \mu \mathrm{m}$ flux density at the location of the southeastern radio source of $15 \mathrm{mJy}$ is $1 / 10$ that of the northern source. This limit, coupled with the $8.4 \mathrm{GHz}$ flux density of this source of $3.2 \mathrm{mJy}$ (C91), leads to a limit on the ratio of

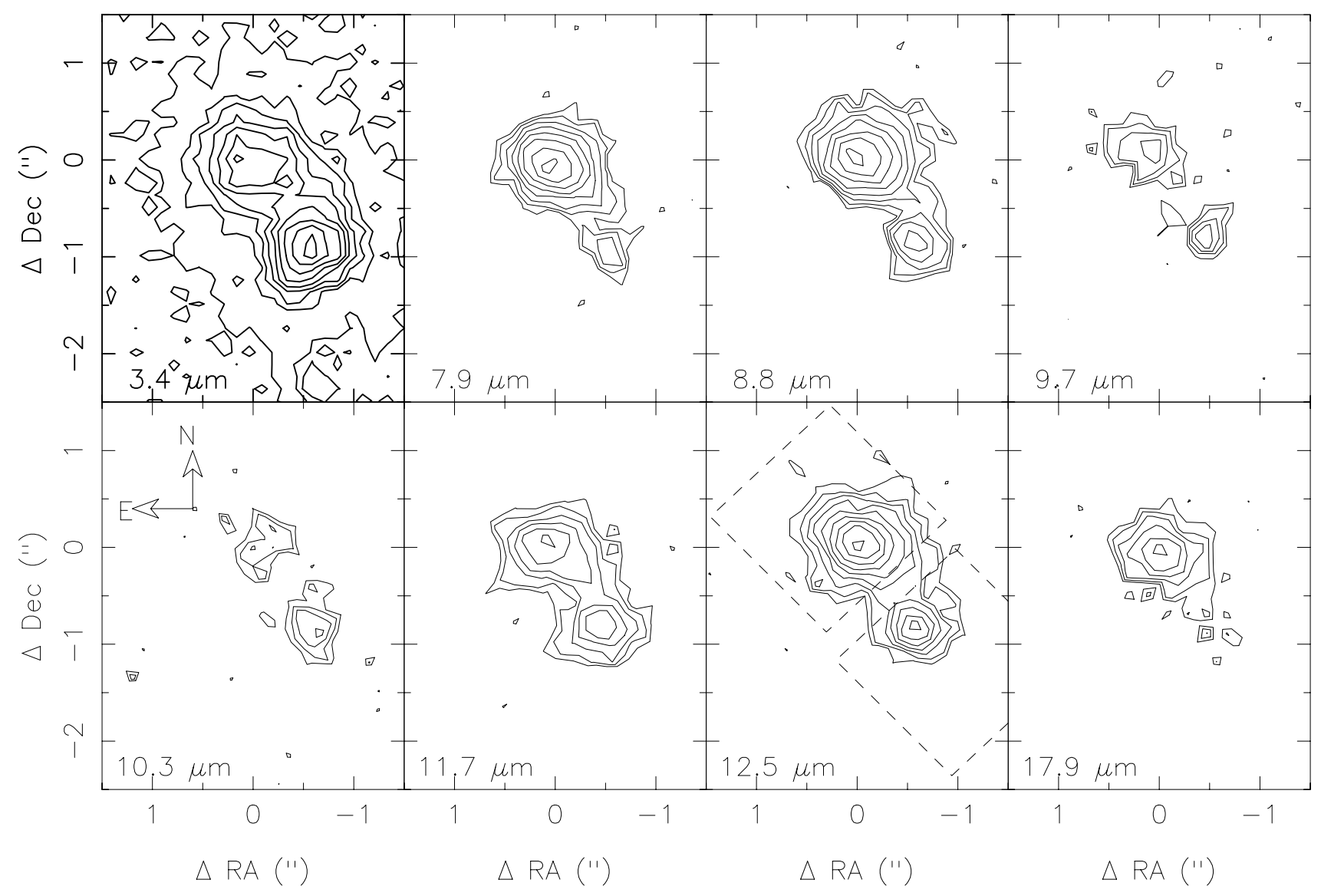

FIG. 4.- Montage of images of Markarian 273. The images at wavelengths from 7.9 to $17.9 \mu \mathrm{m}$ were obtained with MIRLIN. The image at $3.4 \mu \mathrm{m}$ was obtained on the 200 inch Hale Telescope using the Cassegrain infrared camera. The images are centered so that the northern peak is at the location 0,0 in each panel. The locations of the boxes in the $12.5 \mu \mathrm{m}$ image show where the photometry of the peaks was obtained in all the images. 
flux densities $f_{v}(12.5 \mu \mathrm{m}) / f_{v}(8.4 \mathrm{GHz})<4.7$, consistent with the observed ratio $f_{v}(12.5 \mu \mathrm{m}) / f_{v}(8.4 \mathrm{GHz})=4.5$ for the northern source. By comparison, if the radio $(0.2 \mathrm{mJy}$ at 8.4 $\mathrm{GHz}$; C91; Knapen et al.) and infrared (58 mJy at $12.5 \mu \mathrm{m})$ flux densities of the southwestern source are assumed to be associated with the same physical source, the ratio $f_{v}(12.5$ $\mu \mathrm{m}) / f_{v}(8.4 \mathrm{GHz})=290$, a factor of 65 times greater than that seen in the northern source, making this nucleus far more infrared bright relative to the radio than the northern source. If the southwestern radio and infrared sources are not related, the southwestern infrared source has an even larger $f_{v}(12.5 \mu \mathrm{m}) / f_{v}(8.4 \mathrm{GHz})$ ratio.

As can be seen in Figures $1 e$ and 4, the relative brightnesses of the nuclei change substantially as a function of wavelength. In order to get an estimate of the relative flux emitted by the two nuclei, the flux in two identically sized square beams was measured at the same locations on the images at all wavelengths presented; the beams, 1.6 on a side, are shown in Figure 4. The sky was taken northwest and southeast of each of the squares. The efficiency of the square beams was estimated by placing the PSF of the appropriate wavelength at the locations of the nuclei within the square beam. The photometry of the NICMOS image was arbitrarily normalized so that the sum of the fluxes from the two squares equals the sum of the fluxes give by Knapen et al. (1997) and the flux density in a 4" diameter beam equals that of Knapen et al. The results of these photometric measurements are shown in Figure 5.

At 3.4 and $10.3 \mu \mathrm{m}$ the southwestern source is slightly $(10 \%)$ brighter than the northern source. At the other midinfrared wavelengths the northern source is between a factor of 1.5 and 3 times brighter than the southwestern source. The different SEDs of the components implied by their changing relative brightnesses is shown directly in Figure 5, where photometry of the two nuclei is shown. The northern source clearly shows a significantly deeper absorption at $10 \mu \mathrm{m}$ than the southwestern source. This is consistent with the distribution of $\mathrm{CO}$ emission, which strongly

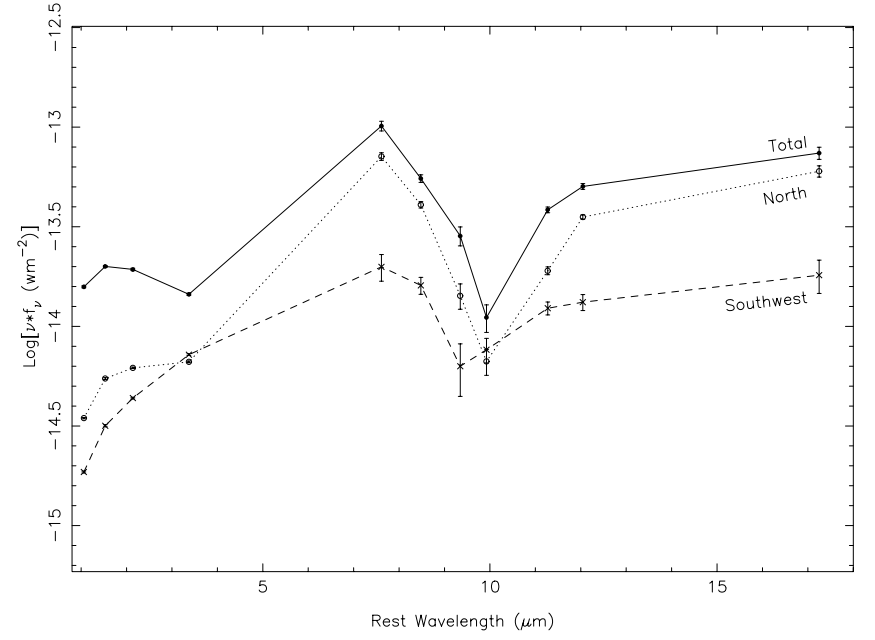

FIG. 5.-Spectral energy distributions of the separate peaks in Markarian 273 as determined from the images presented in Fig. 4. The total flux (also presented in Fig. $3 b$ ) was measured in a 4" diameter beam centered between the peaks and was distributed between the two sources by doing photometry in nonoverlapping boxes (displayed in the $12.5 \mu \mathrm{m}$ panel of Fig. 4) centered on the two peaks. The uncertainties shown are statistical. The systematic uncertainty depends on the wavelength but is probably in the range $15 \%-20 \%$. peaks on the northern source (Downes \& Solomon 1998), but it is inconsistent with the near-infrared colors found by S99, where the northern source has a smaller $J-K$ color than does the southwest source.

The SED of Figure 5, particularly the relatively strong emission at 3.5 and $11.7 \mu \mathrm{m}$, suggests aromatic hydrocarbon emission (PAH) emission associated with the southwest nucleus. Such emission is seen in the spectra of many ULIRGs (Genzel et al. 1998). The emission from the southwest source could account for all of the $11.3 \mu \mathrm{m}$ emission seen in the spectrum of the combined nuclei by Dudley (1999). The northern source does not show a similar peak at $11.7 \mu \mathrm{m}$, but it clearly shows the effects of substantial overlaying cold silicate absorption.

The MIRLIN photometry shown in Figure 3 was obtained using a $4^{\prime \prime}$ diameter beam centered between the two nuclei. The comparison of the MIRLIN and IRAS data (Fig. 3) demonstrates that the mid-infrared emission in this source emerges virtually entirely from the central region. Because of the proximity of the two nuclei and the fairly low signal-to-noise ratio of the nuclei in some of the images, it is difficult to cleanly determine the sizes of the two sources. The data suggest that both nuclei have unresolved midinfrared cores with sizes less than 0"4. In the northern nucleus approximately $\frac{2}{3}$ of the $12.5 \mu \mathrm{m}$ flux is unresolved, while in the southwestern nucleus, about half the flux is unresolved.

$$
\text { 4.6. } \operatorname{Arp} 220=U \text { GC } 9913=\operatorname{IRAS} 15328+2340
$$

The data for this source were discussed in detail in Soifer et al. (1999) and are included in Figures $1 f$ and 3 for completeness.

\subsection{IRAS $17208-0014$}

MIRLIN imaging was obtained at 7.9 and $12.5 \mu \mathrm{m}$. The $12.5 \mu \mathrm{m}$ image is shown in Figure $1 \mathrm{~g}$. The $2.2 \mu \mathrm{m}$ NICMOS image of IRAS $17208-0014$ (Figure $1 g$; S99) shows it to be an extended source of roughly $2^{\prime \prime}$ diameter with a number of luminous starburst centers to the northeast of the nucleus. The mid-infrared image is apparently also similar, but the limitation due to the chop-nod spacing and the relatively low infrared surface brightness results in an image with low signal-to-noise ratio. The size of the $12.5 \mu \mathrm{m}$ source of $\sim 2^{\prime \prime}$ or $1.6 \mathrm{kpc}$ FWHM (Fig. 2) is quite similar as well to that found in CO by Downes \& Solomon (1998) and Jogee et al. (2000).

The best illustration of the spatial distribution of the midinfrared emission is shown in Figure 2. The source flux smoothly increases up to $5^{\prime \prime}$ diameter, with a FWHM of 2"; the apparent leveling off of the enclosed flux at a diameter of $5^{\prime \prime}$ is caused by the artificial imposition of the sky level between diameters of $5^{\prime \prime}$ and $6^{\prime \prime}$.

Evidence that the source is not extended much beyond a diameter of $5^{\prime \prime}$ is provided by Figure 3, which shows that, within the uncertainties, the enclosed flux within a 4" diameter beam is the same as within the IRAS beam. The lack of a dominant compact component in the mid-infrared make this object very different from the other ULIRGs in this sample.

The radio emission from IRAS $17208-0014$ is much more compact than the infrared emission. At least $90 \%$ of the $1.4 \mathrm{GHz}$ flux comes from a region smaller than $2^{\prime \prime} \times 2^{\prime \prime}$ (FWHM, Condon et al. 1996). Martin et al. (1989) measure a radio source with a size of $0.26 \times 0.32$ at $1.6 \mathrm{GHz}$ that 
accounts for $\sim 90 \%$ of the $1.4 \mathrm{GHz}$ flux density, suggesting a compact radio core. This core is clearly not dominant in the mid-infrared imaging. The near-infrared NICMOS imaging of this galaxy (S99) suggests that the nucleus could be obscured at $2.2 \mu \mathrm{m}$, but the lack of detection of a strong compact core at $12.5 \mu \mathrm{m}$ is quite peculiar, since the radio source is so compact.

\section{DISCUSSION}

\subsection{Compact Sources in ULIRGs}

The data presented here, with angular resolution of less than 0.3 , represent the highest spatial resolution achieved to date in probing the central regions of ULIRGs in the thermal infrared. Table 4 summarizes the key observations, the sizes of the compact sources found in the ULIRGs at $12.5,17.9$, and $24.5 \mu \mathrm{m}$, as well as the fraction of the total flux at these wavelengths emitted from these cores. All of the ULIRGs except IRAS $17208-0014$ have a nuclear source in the mid-infrared of size $\leq 200 \mathrm{pc}$ that produces a substantial fraction, if not all, of the galaxy's mid-infrared luminosity. In three sources, IRAS 05189-2524, IRAS $08572+3915$ and Markarian 231, a single unresolved nucleus produces between $80 \%$ and $100 \%$ of the total midinfrared luminosity. In Markarian 273 and Arp 220, two nuclei produce nearly all the mid-infrared emission in the galaxies. In the case of Markarian 273 the nuclei are substantially unresolved, while in Arp 220 at least one nucleus is resolved. Because of its low redshift the linear size of the resolved nucleus in Arp 220 is smaller than the limit on all the unresolved nuclei except Markarian 231 (see Table 5). In only one compact system, UGC 5101, does the compact nucleus produce significantly less than half the total midinfrared emission from the system.

As noted previously, IRAS $17208-0014$ presents an interesting puzzle. There is no mid-infrared evidence for a compact nuclear source, but the radio data (C91; Martin et al. 1989) strongly suggest a power source with a size less than 0 .3. In the following discussion, we will adopt the morphology implied by the $12.5 \mu \mathrm{m}$ image, but we note that extremely high $12.5 \mu \mathrm{m}$ extinction could be leading to an erroneous interpretation of the nuclear structure in IRAS $17208-0014$.

Table 5 shows that the compact nuclear sources have linear sizes typically less than 200 pc in diameter. In Arp 220 the diameter of the western source is $140 \mathrm{pc}$ at $24.5 \mu \mathrm{m}$ (Soifer et al. 1999), while in Markarian 231, the diameter of the $12.5 \mu \mathrm{m}$ source is less than $100 \mathrm{pc}$.

\subsection{AGNs as the Luminosity Source in ULIRGs}

One of the goals of the observations reported here is to distinguish the ultimate source of the infrared luminosity in these systems by means of the spatial extent of the infrared emission. If the dust is heated by a bright compact object such as an AGN, the dust emission will be compact, and the size of the infrared source will increase with increasing wavelength roughly as $r \sim \lambda^{2.5}$, since the dust temperature will decrease with radius as $T \sim r^{-0.4}$ and the wavelength of maximum emission $\lambda_{\max } \sim T^{-1}$. If, on the other hand, the heating is due to many hot stars in a starburst region, then the dust emission will be distributed on the same scale as the young, luminous stars. Since the dust heating is due to the proximity of the dust to the luminous young stars, there will be little or no temperature gradient seen. Spatially extended $10 \mu \mathrm{m}$ emission therefore implies starburst activity, while spatially compact $10 \mu \mathrm{m}$ emission could be due to either a compact starburst or an AGN.

To quantify the compact mid-infrared emission we utilize the brightness temperatures derived from our observations and the color temperatures determined from the IRAS 25 and $60 \mu \mathrm{m}$ observations of these objects. We assume that $T_{c}$ measures the temperature of the grains heated by the luminosity source; $T_{b}$ should be $\leq T_{c}$ if the optical depth $\tau_{v}<1$. Only in the limit of large optical depth should the brightness temperature approach the color temperature.

Based on the MIRLIN observations of Arp 220, Soifer et al. (1999) showed that the dominant luminosity source, Arp $220 \mathrm{~W}$, is optically thick with a brightness temperature at $25 \mu \mathrm{m}$ equal to the color temperature between 25 and $60 \mu \mathrm{m}$. The observations reported here permit analogous constraints to be placed on some of the nuclear sources in other ULIRGs in this sample. In Markarian 231, IRAS $05189-2524$, and IRAS $08572+3915$, the limits on the sizes of the central infrared sources are sufficiently small that it is worthwhile considering how these sizes constrain the far-infrared sources.

The size limit on Markarian 231 of 0 ".13 at $12.5 \mu \mathrm{m}$ places the most severe limits on models of the infrared emitting region. In this case the observed flux density and size limit lead to a lower limit on the brightness temperature of $T_{b} \geq$ $141 \mathrm{~K}$ at $12.5 \mu \mathrm{m}$. If the infrared emitting source has the same size at 17.9, 25, and $60 \mu \mathrm{m}$, the corresponding brightness temperatures are $124 \mathrm{~K}, 128 \mathrm{~K}$ and $241 \mathrm{~K}$. The brightness temperatures at $12.5,17.9$, and $25 \mu \mathrm{m}$ are consistent with each other and with a nearly optically thick source over this wavelength range, but the $60 \mu \mathrm{m}$ brightness temperature is much too large to be consistent with dust models with reasonable grain properties. This implies that the 60 $\mu \mathrm{m}$ source size is much larger than the $12.5 \mu \mathrm{m}$ size.

The color temperature measured between 25 and $60 \mu \mathrm{m}$, determined from the $I R A S$ measurements, is $85 \mathrm{~K}$. The size of a blackbody of $85 \mathrm{~K}$ required to produce the observed 60 $\mu \mathrm{m}$ flux density is 0.4 , significantly larger than the limit on the source size at $12.5 \mu \mathrm{m}$. The source size determined if $T_{c}=T_{b}$ represents a lower limit on the size of the $60 \mu \mathrm{m}$ source. For example, if the size of the $60 \mu \mathrm{m}$ source were equal to the $\sim 1^{\prime \prime}$ size found in $\mathrm{CO}$ by Downes $\&$ Solomon (1998), the $60 \mu \mathrm{m}$ brightness temperature would be $53 \mathrm{~K}$, much less than that inferred by assuming all the 25 and 60 $\mu \mathrm{m}$ emission is emerging from the same region. The size limits are based on the assumption of large optical depth $(\tau \ll 1)$; smaller optical depth would imply an even larger source. Since significant emission is seen at $\lambda<60 \mu \mathrm{m}$, either $\tau_{60 \mu \mathrm{m}}<1$ or we are viewing a tilted optically thick disk, as suggested for Arp 220 (Scoville et al. 1998; Downes \& Solomon 1998; Sakamoto et al. 1999; Soifer et al. 1999). In either case, the size constraints show that in Markarian 231 the infrared source increases in size with increasing wavelength. This is consistent with the picture of an AGN with luminosity of $3 \times 10^{12} L_{\odot}$ heating the surrounding dust and producing a radial temperature gradient.

In this sample there are two additional ULIRGs where significantly more than $50 \%$ of the mid-infrared luminosity emerges from a compact central source, IRAS 05189-2524 and IRAS $08572+3915$. In IRAS $05189-2524$, if the size of the $60 \mu \mathrm{m}$ emitting source is the same as the limit on the $12.5 \mu \mathrm{m}$ source measured here, $T_{b}(60 \mu \mathrm{m})=101 \mathrm{~K}$, which is significantly greater than $T_{c}=85 \mathrm{~K}$ from the IRAS 25 and 
$60 \mu \mathrm{m}$ data. For the same assumptions, in IRAS $08572+3915 T_{b}(60 \mu \mathrm{m})=77 \mathrm{~K}$ is slightly less than $T_{c}=83 \mathrm{~K}$ determined from the $I R A S$ data at 25 and $60 \mu \mathrm{m}$. For both sources, using dust properties described by Draine \& Lee (1984) the sizes calculated for silicate and graphite dust grains heated by central sources of the observed bolometric luminosity and having dust temperatures equal to the observed color temperatures are larger by a factor of 1.5-3 than the observed sizes of these sources at $12.5 \mu \mathrm{m}$. This suggests that the sizes of these infrared sources also increase with increasing mid- and far-infrared wavelength.

High apparent surface brightnesses and a radial temperature gradient are natural consequences of centrally heated dust emission surrounding a high luminosity source, i.e., an AGN. In Markarian 231 there is direct evidence that the size of the infrared source increases with increasing wavelength in the mid- and far-infrared. In IRAS 05189-2524 and IRAS 08572+3915, there is strong evidence for such radial temperature gradients based on the compact sources observed in the mid-infrared. The observed luminosities are adequate to quantitatively account for the inferred variation of dust temperature with radius if the dust is heated by a central source in these objects.

\subsection{Starbursts as the Luminosity Source in ULIRGs and Energy Production Rates}

The compact nature of the sources in the objects observed leads to very high luminosities produced in small volumes. By comparing these energy production rates with those found in local examples of starburst environments, we can determine whether known starburst environments can generate the apparent energy production in the ULIRGs.

In the core of Orion the luminosity of $2 \times 10^{5} L_{\odot}$ emerges from a 0.3 pc size region (Werner et al. 1976), while in the nuclear starburst in M82, the nearest starburst galaxy, the luminosity of $3 \times 10^{10} L_{\odot}$ is emitted over a projected area of $450 \times 75$ pc $\left(30^{\prime \prime} \times 5^{\prime \prime}\right)$ (Rieke et al. 1980). These observations lead to apparent surface brightnesses of emergent luminosity of $1-3 \times 10^{12} L_{\odot} \mathrm{kpc}^{-2}$. If, as seems likely, M82 is being viewed edge-on, then the mean surface brightness of this system, if viewed face-on, is closer to $2 \times 10^{11} L_{\odot} \mathrm{kpc}^{-2}$.

A survey of the maximum surface brightness in nearby starburst galaxies in the UV, infrared, and radio by Meurer et al. (1997) leads to peak global averaged surface brightnesses in starburst galaxies of $\sim 2 \times 10^{11} L_{\odot} \mathrm{kpc}^{-2}$. Meurer et al. find that the peak surface brightnesses of clusters in starburst galaxies reaches $\sim 5 \times 10^{13} L_{\odot} \mathrm{kpc}^{-2}$ over regions $\sim 10 \mathrm{pc}$ in diameter. Meurer et al. suggest that the global peak surface brightnesses is due to a mechanism whereby the pressure from stellar winds and supernovae is able to regulate the star formation rates in galaxy disks, while the formation of massive stars in giant $\mathrm{H}$ II regions is regulated by the "Kennicutt Law;" i.e., star formation occurs when the surface gas density exceeds a critical value that depends on the rotational velocity and shear (Kennicutt 1989).

The surface brightnesses observed in the ULIRGs can be estimated if we assume that the size (or limit) of the region measured in the mid-infrared is representative of the infrared emitting region as a whole. With this assumption, we have presented the surface brightnesses for the observed ULIRGs in Table 5. We include two values of the surface brightness, a "maximum" that assumes that the fraction of the total infrared luminosity emerging from the compact core is the same as the fraction of the mid-infrared flux density emerging from the core, and a "minimum" that assumes that only the observed mid-infrared luminosity emerges from the compact core. In the case of Markarian 231 , the former number is unphysically large ( $\$ 5.2)$. In the other systems, the two values represent likely upper and very conservative lower limits on the apparent surface brightness of the infrared emission.

Only in the case of IRAS 17208-0014, where the near and mid-infrared observations suggest the luminosity is due to star formation, is the measured infrared surface brightness comparable to the peak global value $2 \times 10^{11} L_{\odot}$ $\mathrm{kpc}^{-2}$ found by Meurer et al. (1997). Other than this case, the inferred surface brightnesses are much greater than found globally in disk starburst systems. The highest apparent surface brightnesses in the ULIRGs are found in the western nucleus of Arp 220, which is most likely powered by a nuclear starburst (Smith et al. 1998b) and the cores of Markarian 231 and IRAS 05189-2524, which are probably powered by AGN. In these cores the apparent surface brightness approaches or surpasses the surface brightness of clusters in starburst galaxies, while in the other systems (excluding IRAS 17208-0014) the surface brightness most probably exceeds $10^{13} L_{\odot} \mathrm{kpc}^{-2}$. In all these cases the surface brightness over regions of hundreds of parsecs across are comparable to the brightness found in $\sim 10 \mathrm{pc}$ sized clusters within starburst systems by Meurer et al.

For the ULIRGs that are powered by star formation, the large surface brightnesses and sizes suggest that the global starburst intensity regulating mechanism of Meurer et al. (1997) does not apply. Gravitational instability allows for higher gas densities and hence higher star formation rates in the solid-body (no shear) rotation nuclear regions. Since the nuclei of most ULIRGs are mergers, far from equilibrium, the gas densities may be very high, leading to high star formation rates and correspondingly high surface brightnesses for short times. ULIRGs are clearly extraordinary events in the evolution of galaxies. Several studies (e.g., Sanders et al. 1988; Scoville, Yun, \& Bryant 1997; Downes \& Solomon 1998) suggest that the nuclear disks are unstable in some ULIRGs. The large gas masses in these environments might then lead to nuclear star formation rates much greater than those found in less extreme systems.

\subsection{A Panchromatic View of ULIRGS}

Table 6 summarizes the characterization of the ULIRGs applying many different diagnostics of the underlying energy sources. Here we have summarized the structure in the mid-infrared from the data presented here, in the nearinfrared from S99 and Surace \& Sanders (1999) and in the radio from the VLBI data of Smith et al. (1998a). The optical spectral classifications are from Sanders et al. (1988) and Veilleux et al (1995). The near-infrared spectral classifications are from Veilleux, Sanders, \& Kim (1999) and T. Murphy (1999 private communication). The ISO spectral classifications are based on the strength of the PAH features and are taken from Genzel et al. (1998) and Lutz, Veilleux, \& Genzel (1999). The X-ray classifications are from $A S C A$ results and are taken from Iwasawa (1999) and Nakagawa et al. (1999).

In none of the objects in this sample, excepting perhaps Markarian 273 and IRAS 17208-0014, is there a com- 
TABLE 6

SUMMARY OF PROPERTIES OF ULIRGs

\begin{tabular}{|c|c|c|c|c|c|}
\hline \multirow[b]{2}{*}{ ОВЈЕСт } & \multirow{2}{*}{$\begin{array}{l}\text { COMPACT CORE? } \\
\text { MIR/NIR/RADIO }\end{array}$} & \multicolumn{4}{|c|}{ Spectral Class } \\
\hline & & Optical & Near-IR & ISO & $A S C A$ \\
\hline IRAS $05189 \ldots \ldots$ & $\mathrm{Y} / \mathrm{Y} / \mathrm{N}$ & Sey2 & Sey & $\cdots$ & AGN \\
\hline IRAS $08572 \ldots \ldots$ & $\mathrm{Y} / \mathrm{Y} / \mathrm{N}$ & LINER & $\mathrm{H}$ II & $\cdots$ & SB \\
\hline UGC $5101 \ldots \ldots$ & $\mathrm{Y} / \mathrm{Y} / \mathrm{Y}$ & LINER & $\ldots$ & SB & SB \\
\hline Mrk $231 \ldots \ldots \ldots$ & $\mathrm{Y} / \mathrm{Y} / \mathrm{Y}$ & Sey1 & Sey1 & AGN & SB? \\
\hline Mrk $273 \ldots \ldots \ldots$ & $\mathrm{Y} / \mathrm{Y} / \mathrm{Y}$ & Sey2 & Sey? & AGN & AGN \\
\hline Arp $220 \ldots \ldots \ldots$ & $\mathrm{Y} / \mathrm{N} / \mathrm{Y}$ & LINER & $\ldots$ & SB & SB? \\
\hline IRAS $17208 \ldots \ldots$ & $\mathrm{N} / \mathrm{N} / ?$ & $\mathrm{H}$ II & $\mathrm{H}$ II & SB & $\ldots$ \\
\hline
\end{tabular}

pletely consistent set of "classifications" based on the wide range of wavelengths over which these objects are observed. In the object that is the clearest case for a central AGN luminosity source, Markarian 231, the hard X-ray emission is not consistent with the simplest view of such power sources (Nakagawa et al. 1999). In the predominantly Seyfert nucleus IRAS $05189-2524$ there is not a dominant compact radio core. In Markarian 273 the two nuclei clearly seen in high resolution imaging complicates the classification, since none of the spectral classifications resolved the separate nuclei. In UGC 5101, another source where the optical, infrared, and X-ray indications point toward a starburst power source, a compact radio core characteristic of a central AGN exists. Compact mid-infrared sources occur in six of seven of these sources, where other evidence points strongly to both AGN and starbursts as the dominant underlying power source. Table 6 shows that most ULIRGs do not present a picture over all wavelengths consistent exclusively with either an AGN or starburst power source. In most objects observed here, it is likely that both contribute to some extent to their total bolometric luminosity, though Markarian 231 and IRAS 17208-0014 appear to represent the extremes of dominant AGN and starburst sources.

\section{SUMMARY AND CONCLUSIONS}

Diffraction-limited mid-infrared observations of a sample of seven ULIRGs obtained on the Keck $10 \mathrm{~m}$ telescopes have shown the following:

1. Extremely compact structures, corresponding to emission on spatial scales of less than 100-200 pc, are seen in six of the seven systems observed. The mid-infrared emission in these galaxies generally emerges from a single region of diameter less than $1 \mathrm{kpc}$, and in most cases from regions less than $200 \mathrm{pc}$ in diameter.

2. The upper limit on the diameter of the $12.5 \mu \mathrm{m}$ source in Markarian 231, 0 .'13, is too small to be consistent with the observed $60 \mu \mathrm{m}$ flux density emerging from a region this size and demonstrates that in this AGN the source size increases with increasing wavelength (decreasing dust temperature).

3. In IRAS $05189-2524$ and IRAS $08572+3915$ there is strong circumstantial evidence that the size of the infrared source increases with wavelength between 12.5 and $60 \mu \mathrm{m}$, suggesting that these too are predominantly $A G N$ powered ULIRGs.

4. In the other objects observed, the angular size limits for the compact mid-infrared sources are not yet adequate to distinguish between AGN and starburst luminosity sources, although the small sizes are consistent with a central luminosity source heating the surrounding dust as in AGN models in all the sources except IRAS 17208-0014.

5. If star formation powers the luminosity in the very compact infrared sources seen in these systems, the star formation rates averaged over a few hundred parsecs is comparable to that seen in the brightest $\sim 10$ pc sized clusters in nearby starbursts, and exceeds by factors of up to 100 , the global star formation rates in nearby starburst galaxies.

We thank J. Aycock and R. Campbell for assistance with the observations, R. Goodrich, R. Moskitis, and the entire Keck staff for their help establishing the visitor port and to Barbara Jones, Rick Puetter, and the Keck team that brought the LWS into service, enabling these observations. The W. M. Keck Observatory is operated as a scientific partnership between the California Institute of Technology, the University of California, and the National Aeronautics and Space Administration. It was made possible by the generous financial support of the W. M. Keck Foundation. This research has made use of the NASA/IPAC Extragalactic Database which is operated by the Jet Propulsion Laboratory, Caltech under contract with NASA. B. T. S., G. N., K. M., and E. E. are supported by grants from the NSF and NASA. J. A. S. and B. T. S. are supported by the SIRTF Science Center at Caltech. SIRTF is carried out at the Jet Propulsion Laboratory. E. E. B., A. J. W., N. Z. S., and A. S. E. were supported by NASA grant NAG5-3042. This work was carried out in part (M. W. W., M. R.) at the Jet Propulsion Laboratory, operated by the California Institute of Technology, under an agreement with NASA. The development of MIRLIN was supported by NASA's Office of Space Science. The National Radio Astronomy Observatory (J. J. C.) is a facility of the National Science Foundation operated under cooperative agreement by Associated Universities, Inc. 
REFERENCES

Barger, A. J., Cowie, L. L., Sanders, D. B., Fulton, E., Taniguchi, Y., Sato, Y., Kawara, K., \& Okuda, H. 1998, Nature, 394, 248

Blain, A. W., Smail, I., Ivision, R. J., \& Kneib, J.-P. 1999, MNRAS, 302, 632

Boksenberg, A., Carswell, R. F., Allen, D. A., Fosbury, R. A. E., Penston, M. V., \& Sargent, W. L. W. 1977, MNRAS, 178, 451

Carico, D. P., Sanders, D. B., Soifer, B. T., Elias, J. H., Matthews, K., \& Neugebauer, G. 1988, AJ, 95, 356

Carico, D. P., Graham, J. R., Matthews, K., Wilson, T. D., Soifer, B. T., Neugebauer, G., \& Sanders, D. B. 1990, ApJ, 349, L39

Condon, J. J., Huang, Z. P., Yin, Q. F., Thuan, T. X. 1991, ApJ, 378, 65 (C91)

Condon, J. J., Helou, G., Sanders, D. B., \& Soifer, B. T. 1996, ApJS, 103, 81

Downes, D., \& Solomon, P. M. 1998, ApJ, 507, 615

Draine, B. T., \& Lee, H. M. 1984, ApJ, 285, 89

Dudley, C. C. 1999, MNRAS, 307, 553

Elias, H., et al. 1982, AJ, 7, 1029

Genzel, R., et al. 1998, ApJ, 498, 579

Heckman, T. 1998, in Ultraluminous Galaxies: Monsters or Babies? (astro-ph9903041)

Iwasawa, K. 1999, MNRAS, 302, 96

IRAS Point Source Catalog, Version 2. 1989, Joint IRAS Science Team (Washington: GPO)

Jogee, S., Bell, G. R., \& Scoville, N. Z. 2000, in preparation

Jones, B., Puetter, R. C. 1993, Proc. SPIE, 1946, 610

Joseph, R. D. 1999, in Ultraluminous Galaxies: Monsters or Babies?, preprint

Keto, E., Ball, R., Arens, J., Jernigan, G., \& Meixner, M. 1992, ApJ, 389, 223

Kennicutt, R. C. 1989, ApJ, 344, 685

Knapen, J. H., Laine, S., Yates, J. A., Robinson, A., Richards, A. M. S., Doyon, R., \& Nadeau, D. 1997, ApJ, 490, L29

Lilly, S. J., Eales, S. A., Gear, W. K., Webb, T. M., Bond, J. R., \& Dunne, L. 1999 , in STScI Workshop, Formation of Galactic Bulges, in press (astroph9903157)

Lutz, D., Veillleux, S., \& Genzel, R. 1999, ApJ, 517, L13

Martin, J. M., Bottinelli, L., Dennefeld, M., Gouguenheim, L., \& Le Squeren, A. M. 1989, A\&A, 208, 39

Meurer, G. R., Heckman, T. M., Lehnert, M. D., Leitherer, C., \& Lowenthal, J. 1997, AJ, 114, 54
Miles, J. W., Houck, J. R., Hayward, T. L., \& Ashby, M. L. N. 1996, ApJ, 465,191

Nakagawa, T., Kii, T., Fujimoto, R., Miyazaki, T., Inoue, H., Ogasaka, Y., Arnaud, K., \& Kawabe, R. 1999, in IAU Symp. 186, Galaxy Interactions at High and Low Redshift, ed. J. E. Barnes \& D. B. Sanders (Dordrecht: Kluwer)

Ressler, M. E., Werner, M. W., Van Cleve, J., \& Choa, H. 1994, Exp. Astron., 3, 277

Rieke, G. H. 1988, ApJ, 331, L5

Rieke, G. H., \& Low, F. J. 1972, ApJ,

Rieke, G. H., Lebofsky, M. J., Thompson, R. I., Low, F. J., \& Tokanaga, A. T. 1980, ApJ, 238, 24

Sakamoto, K., Scoville, N. Z., Yun, M. S., Crosas, M., Genzel, R., \& Tacconi, L. J. 1999, ApJ, 514, 68

Sanders, D. B. 1999, in Ultraluminous Galaxies: Monsters or Babies? (astro-ph9908297)

Sanders, D. B., Soifer, B. T., Elias, J., Madore, B., Matthews, K., Neugebauer, G., \& Scoville, N. 1988, ApJ, 325, 74

Sanders, D. B., Egami, E., Lipari, S., Mirabel, I. F., \& Soifer, B. T. 1995, AJ, 110,1993

Scoville, N. Z., et al. 1998, ApJ, 492, L107

Scoville, N. Z., et al. 1999, AJ, submitted (S99)

Scoville, N. Z., Yun, M. S., \& Bryant, P. M. 1997, ApJ, 484, 702

Smith, H. E., Lonsdale, C. J., \& Lonsdale, C. J. 1998, ApJ, 492, 137

Smith, H. E., Lonsdale, C. J., Lonsdale, C. J., \& Diamond, P. J. 1998b, ApJ, 493, L17

Soifer, B. T., Boehmer, L., Neugebauer, G., \& Sanders, D. B. 1989, AJ, 98, 766

Soifer, B. T., Neugebauer, G., Matthews, K., Becklin, E. E., Ressler, M., Werner, M. W., Weinberger, A. J., \& Egami, E. 1999, ApJ, 513, 207

Soifer, B. T., Sanders, D. B., Madore, B. F., Neugebauer, G., Danielson, G. E., Elias, J. H., Lonsdale, C. J., \& Rice, W. L. 1987, ApJ, 320, 238

Surace, J. R., \& Sanders, D. B. 1999, ApJ, 512, 162

Surace, J. R., Sanders, D. B., Vaca, W. D., \& Veilleux, S. 1998, ApJ, 492, 116

Veilleux, S., Kim, D.-C., Sanders, D. B., Mazzarella, J. M., \& Soifer, B. T. 1995, ApJS, 98, 171

Veilleux, S., Sanders, D. B., \& Kim, D.-C. 1999, ApJ, 522, 139

Werner, M. W., Gatley, I., Becklin, E. E., Harper, D. A., Loewenstein, R. F., Telesco, C. M., \& Thronson, H. A. 1976, ApJ, 204, 402 